\title{
SOBOLEV SPACES AND $\nabla$-DIFFERENTIAL OPERATORS ON MANIFOLDS I: BASIC PROPERTIES AND WEIGHTED SPACES
}

\author{
MIRELA KOHR AND VICTOR NISTOR
}

\begin{abstract}
We study $\nabla$-Sobolev spaces and $\nabla$-differential operators with coefficients in general Hermitian vector bundles on Riemannian manifolds, stressing a coordinate free approach that uses connections (which are typically denoted $\nabla)$. These concepts arise naturally from Partial Differential Equations, including some that are formulated on plain Euclidean domains, such as the weighted Sobolev spaces used to study PDEs on singular domains. We prove several basic properties of the $\nabla$-Sobolev spaces and of the $\nabla$-differential operators on general manifolds. For instance, we prove mapping properties for our differential operators and independence of the $\nabla$-Sobolev spaces on the choices of the connection $\nabla$ with respect to totally bounded perturbations. We introduce a Fréchet finiteness condition (FFC) for totally bounded vector fields, which is satisfied, for instance, by open subsets of manifolds with bounded geometry. When (FFC) is satisfied, we provide several equivalent definitions of our $\nabla$-Sobolev spaces and of our $\nabla$-differential operators. We examine in more detail the particular case of domains in the Euclidean space, including the case of weighted Sobolev spaces. We also introduce and study the notion of a $\nabla$-bidifferential operator (a bilinear version of differential operators), obtaining results similar to those obtained for $\nabla$-differential operators. Bilinear differential operators are necessary for a global, geometric discussion of variational problems. We tried to write the paper so that it is accessible to a large audience.
\end{abstract}

\section{Contents}

1. Introduction 2

Contents of the paper 3

Acknowledgments 4

2. Global differential operators and Sobolev spaces 4

2.1. Notation and convention 4

2.2. Vector bundles and connections 4

2.3. Global, geometric $\nabla$-Sobolev spaces 5

2.4. First properties of $\nabla$-Sobolev spaces 8

2.5. Global, geometric $\nabla$-differential operators $\quad 12$

3. Mixed differential operators and totally bounded vector fields 15

3.1. The Fréchet finite generation condition 15

3.2. Totally bounded vector fields and the (FFC) condition 18

3.3. Bounded mixed differential operators 22

3.4. Equivalent definitions of $\nabla$-Sobolev spaces 22

M.K. has been partially supported by AGC35124/31.10.2018. V.N. has been partially supported by ANR-14-CE25-0012-01.

Manuscripts available from http://iecl.univ-lorraine.fr/Victor.Nistor/

AMS Subject classification (2010): 58J32 (primary), 47L80, 58J05, 58H05, $46 \mathrm{~L} 87$. 


\section{INTRODUCTION}

Even if one is interested only in Partial Differential Equations (PDEs) on Euclidean domains, one is quickly lead to consider also equations on vector bundles on manifolds, as noticed in many earlier works, including [13] and [20]. Sobolev spaces on manifolds are useful, for instance, for the investigation of the weighted Sobolev spaces that arise in the study of PDEs on polyhedral domains. Vector bundles arise when one considers systems, such as the elasticity and Stokes systems. This motivates our interest in Sobolev spaces and differential operators with coefficients in a general Hermitian vector bundle $E \rightarrow M$ on a Riemannian manifold $M$ without boundary.

The main goal of this paper is to provide the first steps in an approach to the study of Sobolev spaces and of differential operators on manifolds that is as independent as possible from local coordinates. This requires us to use connections $\nabla$ instead of derivatives in our definitions. In this paper, as, for instance, in [32], we introduce Sobolev spaces and differential operators using iterations $\nabla^{j}$ of the connection $\nabla$. These objects will thus be called $\nabla$-Sobolev spaces and $\nabla$-differential operators.

We prove a few basic, elementary properties of the $\nabla$-Sobolev spaces and $\nabla$ differential operators such as multiplication properties, mapping and restriction properties, and the independence of these definitions on totally bounded perturbations of the connection $\nabla$. We also recall the connection between weighted Sobolev spaces and the usual Sobolev spaces (but for a conformally equivalent metric) on manifolds.

To obtain more in depth results, we consider the set $\mathcal{W}_{b}(M)$ of vector fields on $M$ all of whose covariant derivatives are bounded. We then say that $M$ satisfies the Fréchet finiteness condition $(\mathrm{FFC})$ if $\mathcal{W}_{b}(M)$ is finitely generated as a Fréchet module over $\mathcal{C}_{\mathrm{b}}^{\infty}(M)$, the space of functions all of whose covariant derivatives are bounded (see Definition 3.7). This condition is somewhat close to $M$ being of bounded geometry and is satisfied if, for instance, $M$ is an open subset of a manifold with bounded geometry. If the (FFC) is satisfied, we provide several additional equivalent definitions of our $\nabla$-Sobolev spaces and $\nabla$-differential operators.

We note that our $\nabla$-Sobolev spaces and $\nabla$-differential operators depend on the choice of $\nabla$. While very many works have been devoted to the role of geometry in the study of the properties of Sobolev spaces and differential operators, much fewer works have been devoted to the issues arising from the twisting of the coefficient bundle. See however the papers on "magnetic Sobolev spaces" by Nguyen, Pinamonti, Squassina, and Vecchi [47] and by Iftimie, Măntoiu, and Purice [35]. From a practical point of view, considering operators with coefficients in a vector bundle has practical applications, as it is a framework that is necessary for the modeling of systems, such as the ones arising in solid or continuum mechanics (Lamé - and elasticity in general - and Stokes and Navier-Stokes systems and their generalizations), as well as in the study of the Hodge-Laplacian. For instance, fluid mechanics 
on a curved space-time in a relativistic setting was considered recently by Disconzi, Ifrim, and Tataru [25] (see also [38]).

The role of the geometry of the underlying manifold in the study of the properties of Sobolev spaces and differential operators was much studied and we cannot do justice to all the people who have worked on the subject; nevertheless, let us mention a few of the most important contributions that have influenced this paper. In an early paper, Aronszajn and Milgram [13] have studied scalar differential operators on general Riemannian manifolds, obtaining, in particular, adjoint and Green-type formulas. The reader will find a lot of useful geometric background material accessible to analysts. Browder [20] has also worked in the scalar case and has studied PDEs on a class of euclidean domains that these days are called "manifolds with boundary and bounded geometry." More recently, Sobolev spaces and differential operators were studied in very many papers, see for instance $[1,2,5,14,22,33,44,51]$ and the references therein. The monographs by Aubin [15], Hebey [32], and Taylor [52] provide even more references. Recently Herbert Amann and his collaborators has started a general program to study maximal regularity and PDEs in general on certain singular spaces that can be modelled by manifolds with boundary and bounded geometry, see, for instance $[2,3,4,26]$. A related program (but with a completely different motivation and mostly devoted to elliptic theory) was pursued by the second named author together with several collaborators, see, for instance $[9,30,42]$. This paper fits into this program of the second named author, but the role of the geometry in the study of $\nabla$-Sobolev and $\nabla$-differential operators will mostly be relegated to the second paper of this series [37], since it takes us too far afield from the results obtained in this paper.

Contents of the paper. In Section 2 we recall some basic definitions concerning global differential operators with smooth coefficients and Sobolev spaces in the context of smooth manifolds and smooth vector bundles. Thus, we recall the definition of global, geometric $\nabla$-Sobolev spaces and of weighted $\nabla$-Sobolev spaces and obtain related multiplication and mapping properties, as well as other basic results. Also in this section, we define the $\nabla$-differential operators and prove additional mapping properties. The next section is devoted to totally bounded vector fields and to differential operators generated by covariant derivatives $\nabla_{X}$. We call them mixed differential operators since they make the connection between the $\nabla$-differential operators and the classical differential operators. In addition, we introduce the Fréchet finiteness condition (FFC) for the set of $\mathcal{C}_{\mathrm{b}}^{\infty}$ vector fields on $M$ as a main tool in the study of mixed differential operators. We show that if such a condition is satisfied, then the mixed differential operators coincide with the $\nabla$-differential operators. We also obtain various properties of mixed differential operators based on (FFC). Among of them, we obtain a finite generation property for the algebra of bounded mixed differential operators. We also describe equivalent definitions of $\nabla$-Sobolev spaces in terms of $\nabla$-differential and mixed differential operators. In the last part of this section, we introduce the notion of $\nabla$-bidifferential operator (a bilinear version of a differential operator), and provide a parallel discussion to that of $\nabla$-differential operators, thus obtaining analogies between the properties of $\nabla$-differential operators and those of $\nabla$-bidifferential operators. Bilinear differential operators are necessary for a global, geometric discussion of variational problems. 
Acknowledgments. We thank Herbert Amann, Bernd Ammann, Nadine Große, Sergiu Moroianu, and Radu Purice for useful discussions.

\section{Global differential operators and Sobolev spaces}

In this section we recall some basic definitions. For simplicity, we shall stay as much as possible in the smooth category: smooth manifolds, smooth vector bundles, smooth coefficients, ... Many of our results, however, extend to operators with suitable lower regularity of the coefficients. More often than not, these extensions to lower regularity coefficients are straightforward, but we leave them for another paper.

2.1. Notation and convention. All along the paper, $n$ is the dimension of the underlying space: $M, \mathbb{R}^{n}, \ldots$, and $\mu$ or $2 m$ are the orders of our differential operators. Also, we shall use the following conventions for the many types of dual spaces that we use. If $T: V \rightarrow W$ is a linear map, then $T^{\prime}: W^{\prime} \rightarrow V^{\prime}$ is its dual, where $V^{\prime}$ is the dual space to $V$. If $V$ and $W$ are complex vector spaces endowed with inner products, then $T^{*}: W \rightarrow V$ is the adjoint of $T: V \rightarrow W$. In case of real vector spaces, instead of the adjoint we have the transpose $T^{\top}: W \rightarrow V$.

2.2. Vector bundles and connections. It will be convenient to use the language of manifolds and vector bundles as in $[13,34,50,52]$, for example, but there are many other possible references. In this paper, we follow [9] to which we refer for concepts not defined here, as well as for some other details. Thus, in the following, $M$ will be a smooth Riemannian manifold without boundary with metric $g$. We shall often consider manifolds that are subsets of some Euclidean space, in which case, they will typically be denoted by $\Omega$, possibly decorated with various subscripts. In this subsection we present known, basic results needed in what follows. See $[15,18,32,36]$ for more background on differential geometry and for the unexplained concepts and results.

The space of smooth sections of a vector bundle $E \rightarrow M$ will be denoted $\mathcal{C}^{\infty}(M ; E)$, whereas the space of those sections that in addition have compact support will be denoted $\mathcal{C}_{\mathrm{c}}^{\infty}(M ; E)$. All the vector bundles considered in this paper will be smooth. As usual, $T M \rightarrow M$ is the tangent bundle to $M$ and $T^{*} M \rightarrow M$ is the cotangent bundle to $M$ (the dual of $T M$ ). Recall that a connection $\nabla=\nabla^{E}$ on $E$ is a first order (linear) differential operator

$$
\nabla^{E}: \mathcal{C}_{\mathrm{c}}^{\infty}(M ; E) \rightarrow \mathcal{C}_{\mathrm{c}}^{\infty}\left(M ; T^{*} M \otimes E\right), \quad \nabla^{E}(f u)=d f \otimes u+f \nabla^{E}(u),
$$

where $f \in \mathcal{C}^{\infty}(M)$ and $u \in \mathcal{C}_{\mathrm{c}}^{\infty}(M ; E)$. (All differential operators in this paper will be linear.) If $X \in \mathcal{C}^{\infty}(M ; T M)$ is a smooth vector field, then $i_{X}: \mathcal{C}_{\mathrm{c}}^{\infty}\left(M ; T^{*} M \otimes\right.$ $E) \rightarrow \mathcal{C}_{\mathrm{c}}^{\infty}\left(M ; T^{*} M \otimes E\right)$ is the contraction with $X$ and we have $\nabla_{X}^{E}:=i_{X} \circ$ $\nabla^{E}$. If $E, F \rightarrow M$ are two vector bundles endowed with connections $\nabla^{E}$ and $\nabla^{F}$ respectively, then we shall endow the tensor product vector bundle $E \otimes F$ with the induced connection: $\nabla_{X}^{E \otimes F}=\nabla_{X}^{E} \otimes 1+1 \otimes \nabla_{X}^{F}$. Let $\tau: E \otimes T^{*} M \otimes F \rightarrow$ $T^{*} M \otimes E \otimes F$ be the natural isomorphism permuting the first two factors. Then $\nabla^{E \otimes F}=\nabla^{E} \otimes 1+\tau \circ\left(1 \otimes \nabla^{F}\right)$, which we shall write, by abuse of notation, in the form

$$
\nabla^{E \otimes F}=\nabla^{E} \otimes 1+1 \otimes \nabla^{F}
$$

We shall proceed similarly with endomorphism bundles. 
Remark 2.1. Let $E$ and $F$ be two complex vector bundles on $M$ endowed with connections. We endow $\operatorname{Hom}(E, F)$ with the induced connection. More precisely, let $\kappa: T^{*} M \otimes \operatorname{Hom}(E ; F) \simeq \operatorname{Hom}\left(E ; T^{*} M \otimes F\right)$ denote the natural isomorphism. Then the connection on $\operatorname{Hom}(E ; F)$ is such that, for all $u \in \mathcal{C}_{\mathrm{c}}^{\infty}(M ; E)$ and $a \in$ $\mathcal{C}_{\mathrm{c}}^{\infty}(M ; \operatorname{Hom}(E ; F))$,

$$
\nabla^{F}(a u)=\kappa \nabla^{\operatorname{Hom}(E ; F)}(a) u+(1 \otimes a) \nabla^{E} u,
$$

The natural morphism $\kappa$ will be omitted from the notation from now on. In particular, this construction for $F=\mathbb{C}$, yields the connection on the dual bundle $E^{\prime}:=\operatorname{Hom}(E ; \mathbb{C})$, where the trivial bundle is endowed with the trivial connection. Thus, if we denote by $\langle\rangle:, E^{\prime} \otimes E \rightarrow \mathbb{C}$ the natural pairing, then the connection $\nabla^{E^{\prime}}$ is such that, for all vector fields $X$ and all smooth, compacted supported sections $u$ and $w$ of the vector bundles $E$ and $E^{\prime}$, we have

$$
\left\langle\nabla_{X}^{E^{\prime}} u, w\right\rangle=X\langle u, w\rangle q-\left\langle u, \nabla_{X}^{E} w\right\rangle .
$$

Whenever there is no danger of confusion, we shall drop the superscripts of the connection, thus write $\nabla=\nabla^{E}$. We shall use the notation

$$
V^{\otimes k}:=\underbrace{V \otimes V \otimes \ldots \otimes V}_{\mathrm{k} \text {-times }} \text { and } V^{\otimes 0}:=\mathbb{C} .
$$

In particular, $T^{* \otimes j} M:=\left(T^{*} M\right)^{\otimes j}$ will denote the repeated tensor products of the cotangent space $T^{*} M$, which appears in the range of the iterated connection map $\nabla^{j}:=\nabla^{T^{* \otimes(j-1)} M \otimes E} \circ \ldots \circ \nabla^{T^{*} M \otimes E} \circ \nabla^{E}: \mathcal{C}_{\mathrm{c}}^{\infty}(M ; E) \rightarrow \mathcal{C}_{\mathrm{c}}^{\infty}\left(M ; T^{* \otimes j} M \otimes E\right)$, where $T^{*} M$ and $T M$ are endowed with the Levi-Civita connection $\nabla=\nabla^{T M}=$ $\nabla^{L C}$. The Levi-Civita connection is the unique torsion-free, metric preserving connection on $T M$, a concept that we recall next.

A hermitian vector bundle $E \rightarrow M$ is a complex vector bundle endowed with a (smoothly varying, sesquilinear) inner product $(\cdot, \cdot)_{E}$. Its bounded sections are denoted $L^{\infty}(M ; E)$. A connection $\nabla=\nabla^{E}: \mathcal{C}_{\mathrm{c}}^{\infty}(M ; E) \rightarrow \mathcal{C}_{\mathrm{c}}^{\infty}\left(M ; T^{*} M \otimes E\right)$ is called metric preserving if, for all $\xi, \eta \in \mathcal{C}_{\mathrm{c}}^{\infty}(M ; E)$, we have

$$
X(\xi, \eta)_{E}=\left(\nabla_{X}^{E} \xi, \eta\right)_{E}+\left(\xi, \nabla_{X}^{E} \eta\right)_{E} .
$$

The space of bounded sections $u$ of $E$ such that all their covariant derivatives $\nabla^{j} u \in \mathcal{C}^{\infty}\left(M ; T^{* \otimes j} M \otimes E\right)$ are also bounded is denoted by $\mathcal{C}_{\mathrm{b}}^{\infty}(M ; E)$. If the curvature of $E$ and all its covariant derivatives are bounded (i.e. if the curvature is in $\mathcal{C}^{\infty}$ ), we shall say that $E$ has totally bounded curvature [9].

2.3. Global, geometric $\nabla$-Sobolev spaces. By dvol we shall denote the induced volume form (that is, measure) on $M$ associated to the metric $g$ on $M$. We then let, as usual,

$$
\|u\|_{L^{p}(M ; E)}:= \begin{cases}\left(\int_{M}\|u(x)\|_{E}^{p} \operatorname{dvol}(x)\right)^{1 / p} & \text { if } 1 \leq p<+\infty \\ \inf _{\operatorname{dvol}(N)=0} \sup _{x \in M \backslash N}\|u(x)\|_{E} & \text { if } p=+\infty\end{cases}
$$

(of course $\|u\|_{L^{\infty}(M ; E)}$ is the essential supremum, ess-sup $\|u(x)\|_{E}$, of $u$ ). As usual, we identify sections of $E$ that coincide outside a set of measure zero to define the $L^{p}$-spaces:

$$
L^{p}(M ; E):=\left\{u: M \rightarrow E \mid\|u\|_{p}<+\infty\right\} / \operatorname{ker}\left(\|\cdot\|_{p}\right) .
$$


2.3.1. Definition of $\nabla$-Sobolev spaces. We now introduce the Sobolev spaces in which we are interested in a global way, as in $[9,32]$. We need to use the in$\operatorname{dex} \nabla$ in their notation since there will be other definitions of Sobolev spaces as well and the definition depends on the choice of the connection (in general). We consider only complex Sobolev spaces, but the real case can be treated similarly.

Definition 2.2. Let $M$ be a Riemannian manifold with metric $g$ and volume form dvol. Let $E \rightarrow \mathbb{C}$ be a finite dimensional, hermitian vector bundle with metric preserving connection $\nabla^{E}$. We extend $\nabla^{E}$ to connections $\nabla$ on the bundles $T^{* \otimes k} M \otimes E, k \in \mathbb{N}$, using the Levi-Civita connection $\nabla^{L C}$ on $T M$ (we omit the superscrit to lighten the notation). Let $s \in \mathbb{N}$. Then

$$
W_{\nabla}^{s, p}(M ; E):=\left\{u \mid \nabla^{j}(u) \in L^{p}\left(M ; T^{* \otimes j} M \otimes E\right), \text { for } 0 \leq j \leq s\right\}
$$

is the order $s, L^{p}$-type $\nabla$-Sobolev space of sections of $E$ (so $W_{\nabla}^{0, p}=L^{p}$ ) with norm

$$
\|u\|_{W_{\nabla}^{s, p}(M ; E)}:=\ell^{p}-\operatorname{norm} \text { of }\left\{\left\|\nabla^{j}(u)\right\|_{L^{p}\left(M ; T^{* \otimes j} M \otimes E\right)}, 0 \leq j \leq s\right\} .
$$

When there is no danger of confusion, we shall write $\|u\|_{L^{p}(M)}$ and even $\|u\|_{L^{p}}$

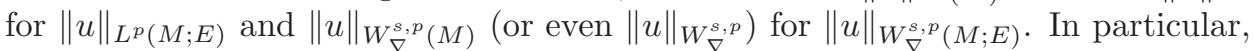
$\|u\|_{W_{\nabla}^{s, \infty}(M)}:=\max _{j=0}^{s}\left\|\nabla^{j}(u)\right\|_{L^{\infty}(M)}$. We let $H_{\nabla}^{s}(M ; E):=W_{\nabla}^{s, 2}(M ; E)$, thus $\|u\|_{H_{\nabla}^{s}(M ; E)}^{2}:=\sum_{j=0}^{s}\left\|\nabla^{j}(u)\right\|_{L^{2}(M)}^{2}$.

As we shall see shortly below, the spaces $W_{\nabla}^{s, p}$ do have some of the usual properties of the Sobolev spaces on compact, smooth manifolds (with or without boundary) provided that $M$ has bounded geometry, see for instance [9, 31, 32, 53] and the references therein. See [27, 28] for related papers using Sobolev spaces that go beyond the bounded geometry setting. The reader should be cautioned, however, not to take everything for granted since, for instance, the spaces $W_{\nabla}^{s, p}$ do depend on the choice of $\nabla$. See, for instance, Example 2.8 on "magnetic Sobolev spaces."

The space

$$
\mathcal{C}_{\mathrm{b}}^{\infty}(M ; E):=W_{\nabla}^{\infty, \infty}(M ; E):=\bigcap_{s \in \mathbb{N}} W_{\nabla}^{s, \infty}(M ; E),
$$

introduced above, that is, the space of bounded sections of $E$ such that all their covariant derivatives are also bounded, will play an important role in what follows. It is a Fréchet space endowed with the increasing family of semi-norms $\|\cdot\|_{W_{\nabla}^{s, \infty}(M ; E)}$. Recall that a subset $S \subset \mathcal{C}_{\mathrm{b}}^{\infty}$ is bounded if, and only if, it is bounded in every semi-norm (see, e.g., [49, Theorem 1.37]).

2.3.2. Negative and non-integer order $\nabla$-Sobolev spaces. Let next $W_{0, \nabla}^{s, p}(M ; E), s \in$ $\mathbb{N}$, be the closure of the space $\mathcal{C}_{\mathrm{c}}^{\infty}(M ; E)$ in $W_{\nabla}^{s, p}(M ; E)$. If $M$ is complete, then, for any $1 \leq p<\infty, W_{0, \nabla}^{1, p}(M ; E)=W_{\nabla}^{1, p}(M ; E)$ (see [32, Theorem 2.7]). Moreover, if $s \in \mathbb{N}, s \geq 2$, and $M$ is complete with positive injectivity radius and Ricci curvature bounded up to the order $s-2$, then, for any $1 \leq p<\infty, W_{0, \nabla}^{s, p}(M ; E)=W_{\nabla}^{s, p}(M ; E)$ (see [32, Theorem 2.8]).

We shall use the spaces $W_{0, \nabla}^{s, p}(M ; E)$, for $1<p<+\infty$, to define the Sobolev spaces with negative index

$$
W^{-s, p}\left(M ; E^{*}\right):=W_{0, \nabla}^{s, p^{\prime}}(M ; E)^{*},
$$

where $V^{*}$ is the complex conjugate dual of $V$ and $\frac{1}{p}+\frac{1}{p^{\prime}}=1$. For simplicity, in the following, we shall identify $E^{*}$ with $E$ using the hermitian metric on $E$, so 
$W^{-s, p}\left(M ; E^{*}\right) \simeq W^{-s, p}(M ; E)$. We also define the spaces $W_{\nabla}^{s, p}(M ; E)$ for $s \notin \mathbb{Z}$ by complex interpolation (see $[41,54]$ for further details).

2.3.3. Weighted $\nabla$-Sobolev spaces. One of the main reason for our interest in general Sobolev spaces on manifolds is that they are useful in the study of weighted Sobolev spaces, whose definition we recall next (compare with Definition 2.2).

Definition 2.3. Let $\rho, f_{0}: M \rightarrow(0, \infty)$ be given. Let $s \in \mathbb{N}$ and $p \in[1,+\infty]$. Then

$$
f_{0} W_{\nabla, \rho}^{s, p}(M ; E):=\left\{u \mid \rho^{j} \nabla^{j}\left(f_{0}^{-1} u\right) \in L^{p}(M ; E), \text { for } 0 \leq j \leq s\right\},
$$

is the order $s, L^{p}$-type $\nabla$-weighted Sobolev space of sections of $E$, (in particular, $\left.f_{0} W_{\nabla, \rho}^{0, p}=f_{0} L^{p}\right)$, endowed with the norm

$$
\|u\|_{f_{0} W_{\nabla, \rho}^{s, p}(M)}:=\ell^{p}-\text { norm of }\left\{\left\|\rho^{j} \nabla^{j}\left(f_{0}^{-1} u\right)\right\|_{L^{p}(M)}, 0 \leq j \leq s\right\} .
$$

We let $f_{0} H_{\nabla, \rho}^{s}(M ; E):=f_{0} W_{\nabla, \rho}^{s, 2}(M ; E)$, and, thus, for any $u \in f_{0} H_{\nabla, \rho}^{s}(M ; E)$, $\|u\|_{f_{0} H_{\nabla, \rho}^{s}(M ; E)}^{2}:=\sum_{j=0}^{s}\left\|\rho^{j} \nabla^{j}\left(f_{0}^{-1} u\right)\right\|_{L^{p}(M)}^{2}$.

These weighted spaces are quite important in applications to geometry $[1,6,28$, $29,30,45]$ or to PDEs $[16,19,23,24,25,39,40]$. Their study is similar to that of the standard (unweighted) Sobolev spaces. One is lead to consider them even if one is interested only on PDEs on domains in $\mathbb{R}^{n}$ and they can be studied by relating them to Sobolev spaces on manifolds. If $f_{0}=\rho=1$, these spaces, of course, reduce to the standard $L^{p}$-type Sobolev space $W_{\nabla}^{s, p}=1 W_{\nabla, 1}^{s, p}$ considered above. Often, the study of weighted Sobolev spaces can be reduced to that of usual Sobolev spaces using conformal changes of metric, see, for instance, $[2,8,7]$.

To illustrate this property, we denote by $g$ the metric tensor of $M$ with the associated Levi-Civita connection $\nabla^{L C}$, and assume that $\rho, f_{0}: M \rightarrow(0, \infty)$ are measurable functions. Let $g_{0}:=\rho^{-2} g$. Assume that $\rho$ is an admissible weight with respect to the metric $g_{0}$ (that is, $\rho$ is smooth and $\rho^{-1} d \rho \in \mathcal{C}_{\mathrm{b}}^{\infty}\left(M ; T^{*} M\right)$ ) and that $f_{0}$ is continuous. Then for $\ell \in \mathbb{N}$ and $p \in[1, \infty]$, we consider the weighted Sobolev space $f_{0} W_{\nabla, \rho}^{\ell, p}(M ; E)$ and the classical Sobolev space $W^{\ell, p}\left(M, g_{0} ; E\right.$ ) (defined with respect to the new metric $\left.g_{0}\right)$. The relation between these spaces is given by the formula

$$
f_{0} W_{\nabla, \rho}^{\ell, p}(M ; E)=f_{0} \rho^{-\frac{n}{p}} W^{\ell, p}\left(M, g_{0} ; E\right), \quad \forall \ell \in \mathbb{N}, p \in[1, \infty]
$$

$[2,11]$, and, especially, Equation (1) in [8] (recall that we are assuming $\rho$ to be admissible). This relation between weighted and unweighted Sobolev spaces provides a strong motivation for our study, even if one is not interested in PDEs on manifolds, since weighted spaces appear naturally in the study of polyhedral domains (thus even in the flat space).

2.3.4. Negative order $\nabla$-weighted Sobolev spaces. Let $\rho, f_{0}: M \rightarrow(0, \infty)$ be given. Let $s \in \mathbb{N}, 1<p<+\infty$, and $f_{0} W_{0, \nabla, \rho}^{s, p}(M ; E)$ be the closure of the space $\mathcal{C}_{\mathrm{c}}^{\infty}(M ; E)$ in $f_{0} W_{\nabla, \rho}^{s, p}(M ; E)$. We use the spaces $f_{0} W_{0, \nabla, \rho}^{s, p}(M ; E)$ to define the weighted Sobolev space with negative index

$$
f_{0} W_{\nabla, \rho}^{-s, p}\left(M ; E^{*}\right):=\left(f_{0}^{-1} W_{0, \nabla, \rho}^{s, p^{\prime}}(M ; E)\right)^{*},
$$

where $\frac{1}{p}+\frac{1}{p^{\prime}}=1$. Recall that we identify $E^{*}$ with $E$ using the hermitian metric on $E$, so $f_{0} W_{\nabla, \rho}^{-s, p}\left(M ; E^{*}\right) \simeq f_{0} W_{\nabla, \rho}^{-s, p}(M ; E)$. 
2.4. First properties of $\nabla$-Sobolev spaces. We now prove a multiplicative property of our Sobolev spaces

Proposition 2.4. Let $\ell \in \mathbb{N}$ and all $p, q, r \in[1, \infty]$ be such that $\frac{1}{p}+\frac{1}{q}=\frac{1}{r}$. Then there is a constant $C_{\ell, p, q}>0$ such that, for all $M$, all $E, E_{1} \rightarrow M$, all connections $\nabla^{E}$ and $\nabla^{E_{1}}$, and all $(a, u) \in W_{\nabla}^{\ell, p}\left(M ; \operatorname{Hom}\left(E ; E_{1}\right)\right) \times W_{\nabla}^{\ell, q}(M ; E)$, we have

$$
\|a u\|_{W_{\nabla}^{\ell, r}\left(M ; E_{1}\right)} \leq C_{\ell, p, q}\|a\|_{W_{\nabla}^{\ell, p}\left(M ; \operatorname{Hom}\left(E ; E_{1}\right)\right)}\|u\|_{W_{\nabla}^{\ell, q}(M ; E)} .
$$

In particular, the evaluation in $E$ defines a continuous bilinear map

$$
W_{\nabla}^{\ell, p}\left(M ; \operatorname{Hom}\left(E ; E_{1}\right)\right) \times W_{\nabla}^{\ell, q}(M ; E) \ni(a, u) \mapsto a u \in W_{\nabla}^{\ell, r}\left(M ; E_{1}\right) .
$$

Proof. Let us assume that at least one of the indices $p$ and $q$, for instance, $p$ belongs to $[1, \infty)$. The case $p=\infty$ is similar, but easier. We show our property by induction on $\ell$. If $\ell=0$ the result is known. Indeed, if $(a, u) \in L^{p}\left(M ; \operatorname{Hom}\left(E ; E_{1}\right)\right) \times$ $L^{q}(M ; E)$, then $a u \in L^{r}\left(M ; E_{1}\right)$, by the multiplication property $L^{p}\left(M ; \operatorname{Hom}\left(E ; E_{1}\right)\right) \times$ $L^{q}(M ; E) \hookrightarrow L^{r}\left(M ; E_{1}\right)$. Moreover, we have

$$
\|a u\|_{L^{r}(M)} \leq\|a\|_{L^{p}(M)}\|u\|_{L^{q}(M)},
$$

and hence the bilinear map is continuous and $C_{0, p, q}=1$ is independent of all choices.

Assume now that our statement is valid for $\ell-1 \geq 0$, and show that it holds also for $\ell$. Let $(a, u) \in W_{\nabla}^{\ell, p}\left(M ; \operatorname{Hom}\left(E ; E_{1}\right)\right) \times W_{\nabla}^{\ell, q}(M ; E)$. Then in view of the embedding $W_{\nabla}^{\ell, p}\left(M ; \operatorname{Hom}\left(E ; E_{1}\right)\right) \times W_{\nabla}^{\ell, q}(M ; E) \hookrightarrow W_{\nabla}^{\ell-1, p}\left(M ; \operatorname{Hom}\left(E ; E_{1}\right)\right) \times$ $W_{\nabla}^{\ell-1, q}(M ; E)$ and by the induction hypothesis we obtain that $a u \in W_{\nabla}^{\ell-1, r}\left(M ; E_{1}\right)$. Thus,

$$
\nabla^{j}(a u) \in L^{r}\left(M ; T^{* \otimes j} M \otimes E_{1}\right), \quad 0 \leq j \leq \ell-1 .
$$

It remains to show that $\nabla^{\ell}(a u) \in L^{r}\left(M ; T^{* \otimes \ell} M \otimes E_{1}\right)$. To this end, we use the formula

$$
\nabla(a u)=\nabla(a) u+(1 \otimes a) \nabla u
$$

(see Remark 2.1) and obtain that

$$
\nabla^{\ell}(a u)=\nabla^{\ell-1}(\nabla(a u))=\nabla^{\ell-1}(\nabla(a) u+(1 \otimes a) \nabla u) .
$$

We have $\nabla a \in W_{\nabla}^{\ell-1, p}\left(M ; \operatorname{Hom}\left(E, T^{*} M \otimes E_{1}\right)\right)$ and $u \in W_{\nabla}^{\ell-1, q}(M ; E)$ and, thus, $\nabla(a) u \in W_{\nabla}^{\ell-1, r}\left(M ; E_{1}\right)$ by the induction hypothesis. The same argument gives that $(1 \otimes a) \nabla u \in W_{\nabla}^{\ell-1, r}\left(M ; E_{1}\right)$. Therefore, $\nabla^{\ell}(a u) \in L^{r}\left(M ; E_{1}\right)$ by formulas $(10)$ and (12) with continuous dependence. Hence $a u \in W_{\nabla}^{\ell, r}\left(M ; E_{1}\right)$ and the induced map is continuous, as asserted. More precisely, for all $p<\infty, q, r \in[1, \infty]$, such that $\frac{1}{p}+\frac{1}{q}=\frac{1}{r}$, we obtain that

$$
\begin{aligned}
\|a u\|_{W_{\nabla}^{\ell, r}(M)}^{r} \leq & \|a u\|_{W_{\nabla}^{\ell-1, r}(M)}^{r}+\|\nabla(a u)\|_{W_{\nabla}^{\ell-1, r}(M)}^{r} \\
\leq & C_{\ell-1, p, q}\|a\|_{W_{\nabla}^{\ell-1, p}(M)}^{r}\|u\|_{W_{\nabla}^{\ell-1, q}(M)}^{r}+\|\nabla(a) u+(1 \otimes a) \nabla u\|_{W_{\nabla}^{\ell-1, r}(M)}^{r} \\
\leq & C_{\ell-1, p, q}\left(\|a\|_{W_{\nabla}^{\ell-1, p}(M)}^{r}\|u\|_{W_{\nabla}^{\ell-1, q}(M)}^{p}+2^{r-1}\left(\|\nabla a\|_{W_{\nabla}^{\ell-1, p}(M)}^{r}\|u\|_{W_{\nabla}^{\ell-1, q}(M)}^{r}\right.\right. \\
& \left.\left.\quad+\|1 \otimes a\|_{W_{\nabla}^{\ell-1, p}(M)}^{r}\|\nabla u\|_{W^{\ell-1, q}(M)}^{r}\right)\right) \\
& \leq C_{\ell-1, p, q}\left(1+2^{r}\right)\|a\|_{W_{\nabla}^{\ell, p}(M)}^{r}\|u\|_{W_{\nabla}^{\ell, q}(M)}^{r}
\end{aligned}
$$




$$
=C_{\ell, p, q}^{r}\|a\|_{W_{\nabla}^{\ell, p}(M)}^{r}\|u\|_{W_{\nabla}^{\ell, q}(M)}^{p},
$$

where $C_{\ell, p, q}^{r}:=C_{\ell-1, p, q}\left(1+2^{r}\right)$.

Corollary 2.5. Let $\ell \in \mathbb{N}$ and all $q \in[1, \infty]$. Then there is a constant $C_{\ell, q}>0$ such that, for all $M$, all $E, E_{1} \rightarrow M$, all connections $\nabla^{E}$ and $\nabla^{E_{1}}$, and all $(a, u) \in$ $W_{\nabla}^{\ell, \infty}\left(M ; \operatorname{Hom}\left(E ; E_{1}\right)\right) \times W_{\nabla}^{\ell, q}(M ; E)$, we have

$$
\|a u\|_{W_{\nabla}^{\ell, q}\left(M ; E_{1}\right)} \leq C_{\ell, q}\|a\|_{W_{\nabla}^{\ell, \infty}\left(M ; \operatorname{Hom}\left(E ; E_{1}\right)\right)}\|u\|_{W_{\nabla}^{\ell, q}(M ; E)} .
$$

In particular, the evaluation in $E$ defines a continuous bilinear map

$$
W_{\nabla}^{\ell, \infty}\left(M ; \operatorname{Hom}\left(E ; E_{1}\right)\right) \times W_{\nabla}^{\ell, q}(M ; E) \ni(a, u) \mapsto a u \in W_{\nabla}^{\ell, q}\left(M ; E_{1}\right) .
$$

Recall that $g$ is a metric of the $n$-dimensional manifold $M$ and $\nabla^{L C}$ is the associated Levi-Civita connection. Assume that $\rho, f_{0}, h_{0}: M \rightarrow(0, \infty)$ are measurable functions. Let $g_{0}:=\rho^{-2} g$ and $\nabla_{0}$ be the associated Levi-Civita connection. Assume that $\rho$ is an admissible weight with respect to the metric $g_{0}$. Let $\operatorname{grad}_{g_{0}} \phi$ denote the vector field which represents the image of $d \phi$ in $T M$ under the metric $g_{0}$. If $\rho=e^{\phi}$ then we have the following the relation between $\nabla^{L C}$ and $\nabla_{0}$ :

$$
\left(\nabla^{L C}-\nabla_{0}\right)_{X} Y=X(\phi) Y+Y(\phi) X-g_{0}(X, Y) \operatorname{grad}_{g_{0}} \phi,
$$

(see, for instance, formula (5) in [10]).

Let $W_{0}^{\ell, p}\left(M, g_{0} ; E\right)$ denote the classical Sobolev space defined as the completion of the space $\mathcal{C}_{\mathrm{c}}^{\infty}(M ; E)$ with respect to the norm given by (4) and corresponding to the metric $g_{0}$. Then formula (8) and Proposition 2.4 applied to the classical Sobolev spaces $W^{\ell, p}\left(M, g_{0} ; E\right)$ lead to the following multiplication property of weighted Sobolev spaces.

Proposition 2.6. Let $\ell \in \mathbb{N}$ and $p, q, r \in[1, \infty]$ be such that $\frac{1}{p}+\frac{1}{q}=\frac{1}{r}$. Let $f_{0}, h_{0}: M \rightarrow(0, \infty)$ be given measurable functions. If $\rho: M \rightarrow(0, \infty)$ is an admissible weight with respect to the metric $g_{0}=\rho^{-2} g$ and $f_{0}, h_{0}$ are continuous, then the bilinear map

$$
f_{0} W_{\nabla, \rho}^{\ell, p}\left(M ; \operatorname{Hom}\left(E ; E_{1}\right)\right) \times h_{0} W_{\nabla, \rho}^{\ell, q}(M ; E) \ni(a, u) \mapsto a u \in f_{0} h_{0} W_{\nabla, \rho}^{\ell, r}\left(M ; E_{1}\right)
$$

is continuous.

Proof. In view of Proposition 2.4 we obtain the continuity of the embedding (of the product of classical Sobolev spaces defined with respect to the metric $g_{0}$ )

$$
W^{\ell, p}\left(M, g_{0} ; \operatorname{Hom}\left(E ; E_{1}\right)\right) \times W^{\ell, q}\left(M, g_{0} ; E\right) \hookrightarrow W^{\ell, r}\left(M, g_{0} ; E\right) .
$$

Then formula 8 implies the continuity of the embedding

$$
\begin{aligned}
f_{0} W_{\nabla^{L C}, \rho}^{\ell, p}\left(M ; \operatorname{Hom}\left(E ; E_{1}\right)\right) \times h_{0} W_{\nabla^{L C}, \rho}^{\ell, q}(M ; E) \\
\quad=f_{0} \rho^{-\frac{n}{p}} W^{\ell, p}\left(M, g_{0} ; \operatorname{Hom}\left(E ; E_{1}\right)\right) \times h_{0} \rho^{-\frac{n}{q}} W^{\ell, q}\left(M, g_{0} ; E\right) \\
\quad \hookrightarrow\left(f_{0} h_{0}\right) \rho^{-\left(\frac{n}{p}+\frac{n}{q}\right)} W^{\ell, r}\left(M, g_{0} ; E\right) \\
\quad=\left(f_{0} h_{0}\right) \rho^{-\frac{n}{r}} W^{\ell, r}\left(M, g_{0} ; E\right) \\
\quad=f_{0} h_{0} W_{\nabla^{L C}, \rho}^{\ell, r}(M ; E),
\end{aligned}
$$

that is, the desired result. 
To understand the complications introduced by the use of the connection, let us discuss the case of "magnetic Sobolev spaces" [47, 35]. Let us introduce first some notation.

Notations 2.7. Assume $M \subset \mathbb{R}^{n}$, so we have global coordinates $x_{j}$. We shall use the following notation

(i) $I:=\{1,2, \ldots, n\}$ and $J_{\mu}:=\{\emptyset\} \cup I \cup I^{2} \cup \ldots \cup I^{\mu}$;

(ii) $\left(e_{j}:=\partial_{j}:=\frac{\partial}{\partial x_{j}}\right)_{j \in I}$ and $\left(e_{j}^{*}:=d x_{j}\right)_{j \in I}$, are the standard basis of $T \Omega$ and, respectively $T^{*} \Omega$, and $e_{\mathbf{i}}:=e_{i_{0}} \otimes e_{i_{1}} \otimes \ldots e_{i_{r}}$ and $e_{\mathbf{i}}^{*}:=e_{i_{0}}^{*} \otimes e_{i_{1}}^{*} \otimes \ldots e_{i_{r}}^{*}$, where $\mathbf{i}=\left(i_{1}, i_{2}, \ldots, i_{r}\right) \in I^{r} \subset J_{\mu}$ and $e_{\emptyset}=e_{\emptyset}^{*}=1 \in \mathbb{C}$;

(iii) For $\mathbf{i}=\left(i_{1}, i_{2}, \ldots, i_{r}\right) \in I^{r} \subset J_{\mu}$, let $|\mathbf{i}|:=r$

(iv) For $\mathbf{i}=\left(i_{1}, i_{2}, \ldots, i_{r}\right) \in I^{r}$, let $\nabla_{\mathbf{i}}:=\nabla_{i_{1}} \nabla_{i_{2}} \ldots \nabla_{i_{r}}$.

Example 2.8 (Magnetic Sobolev spaces). Let us assume that $M=\mathbb{R}^{n}$ with the flat metric, but that the trivial bundle $E=M \times V \rightarrow M$ has a non-trivial connection, where $V$ is some given real vector space. Let $\nabla_{j}=\nabla_{e_{j}}$ be the covariant derivative with respect to the vector field $e_{j}:=\frac{\partial}{\partial x_{j}}$. Then $\nabla_{j}\left(d x_{k}\right)=0$ and

$$
\nabla_{j}(\xi):=\partial_{j} \xi+A_{j} \xi
$$

where $\partial_{j}$ is the partial derivative with respect to $j$ th variable (which is defined since $E \rightarrow M=\mathbb{R}^{n}$ is a trivial bundle) and $A_{j} \in \mathcal{C}^{\infty}(M ; \operatorname{End}(V))$. Recall the notation of 2.7. We agree that $\nabla_{\emptyset} u=u$.

By induction, we then obtain that

$$
\nabla^{r} u=\sum_{\mathbf{i} \in J^{r}} e_{\mathbf{i}}^{*} \otimes \nabla_{\mathbf{i}} u .
$$

We obtain, in particular, that

$$
u \in W_{\nabla}^{r, p}(M ; E) \Leftrightarrow \forall \mathbf{i} \in J_{r}, \nabla_{\mathbf{i}} u \in L^{p}\left(M ; T^{* \otimes|\mathbf{i}|} M \otimes E\right) .
$$

Because of the lack of commutation (in general) of the operators $\nabla_{i}$, these conditions may become more stringent than in the classical case. Let us assume furthermore that $n=2, V=\mathbb{C}^{2}, A_{1}=0$, and

$$
A_{2}:=\left(\begin{array}{cc}
0 & e^{\imath x_{1}^{3}} \\
-e^{-\imath x_{1}^{3}} & 0
\end{array}\right) .
$$

(Notice that $A_{2}^{*}=-A_{2}$.) Then $H_{\nabla}^{1}(M ; E)=H^{1}(M ; E)$, but $H_{\nabla}^{2}(M ; E) \neq$ $H^{2}(M ; E)$. Indeed, let us use Equation (14). We shall write $\nabla_{\mathbf{i}}=\nabla_{(1,1)}=\nabla_{e_{1}}^{2}$ when $\mathbf{i}=(1,1)$, and so on, according to 2.7 . Then we obtain

$$
\begin{aligned}
& \nabla_{(1,1)} \xi=\partial_{1}^{2} \xi, \nabla_{(1,2)} \xi=\partial_{1} \partial_{2} \xi+\left(3 \imath x_{1}^{2} e^{\imath x_{1}^{3}} \xi_{2}+e^{\imath x_{1}^{3}} \partial_{1} \xi_{2}, 3 \imath x_{1}^{2} e^{-\imath x_{1}^{3}} \xi_{1}-e^{-\imath x_{1}^{3}} \partial_{1} \xi_{1}\right) \\
& \nabla_{(2,1)} \xi=\partial_{2} \partial_{1} \xi+\left(e^{\imath x_{1}^{3}} \partial_{1} \xi_{2},-e^{-\imath x_{1}^{3}} \partial_{1} \xi_{1}\right), \nabla_{(2,2)} \xi=\partial_{2}^{2} \xi+2\left(e^{\imath x_{1}^{3}} \partial_{2} \xi_{2},-e^{-\imath x_{1}^{3}} \partial_{2} \xi_{1}\right)-\xi .
\end{aligned}
$$

See $[35,47]$.

We continue with some further properties of $\nabla$-Sobolev spaces.

Corollary 2.9. The tensor product defines a continuous bilinear map

$$
W_{\nabla}^{\ell, \infty}(M ; E) \times W_{\nabla}^{\ell, p}\left(M ; E_{1}\right) \ni(u, v) \mapsto u \otimes v \in W_{\nabla}^{\ell, p}\left(M ; E \otimes E_{1}\right) .
$$

Proof. The natural map $E \rightarrow \operatorname{Hom}\left(E_{1}, E \otimes E_{1}\right)$ is contractive, so the continuity of the map (18) follows from Proposition 2.5. 
Proposition 2.10. Let $E, F \rightarrow M$ be hermitian vector bundles endowed with metric preserving connections. Then the pointwise trace $\operatorname{tr}_{x}: E_{x} \otimes E_{x}^{\prime} \rightarrow \mathbb{C}, x \in M$, $\operatorname{tr}_{x}\left(v^{\prime} \otimes v\right):=\left\langle v^{\prime}, v\right\rangle$, defines the canonical contraction $\epsilon: E \otimes E^{\prime} \otimes F \rightarrow F$, which satisfies $\nabla(\epsilon)=0$. Consequently, $\epsilon \in W^{\infty, \infty}\left(M ; \operatorname{Hom}\left(E \otimes E^{\prime} \otimes F ; F\right)\right)$ and it thus induces a continuous map

$$
\epsilon: W_{\nabla}^{\ell, p}\left(M ; E \otimes E^{\prime} \otimes F\right) \rightarrow W_{\nabla}^{\ell, p}(M ; F) .
$$

Proof. We have $\left\|\operatorname{tr}_{x}\right\| \leq 1$ by the definition of the norm on $E^{\prime}$ (we endow the tensor product with the projective tensor product norm). Consequently $\|\epsilon\|_{\infty} \leq 1$. The relation $\nabla(\epsilon)=0$ follows from the definition of the connection on $E^{\prime}$ and on tensor products. Indeed, using Remark 2.1, we obtain

$$
\begin{aligned}
\nabla(\epsilon)\left(v^{\prime} \otimes v \otimes \xi\right) & =\nabla\left[\epsilon\left(v^{\prime} \otimes v \otimes \xi\right)\right]-\epsilon\left[\nabla\left(v^{\prime} \otimes v \otimes \xi\right)\right] \\
& =\nabla\left(\left\langle v^{\prime}, v\right\rangle \otimes \xi\right)-\epsilon\left[\nabla\left(v^{\prime} \otimes v\right) \otimes \xi+v^{\prime} \otimes v \otimes \nabla(\xi)\right] \\
& =\left[d\left\langle v^{\prime}, v\right\rangle+\epsilon \nabla\left(v^{\prime} \otimes v\right)\right] \otimes \xi+\left\langle v^{\prime}, v\right\rangle \otimes \nabla(\xi)-\epsilon\left(v^{\prime} \otimes v \otimes \nabla(\xi)\right) \\
& =\left[d\left\langle v^{\prime}, v\right\rangle+\left\langle\nabla\left(v^{\prime}\right), v\right\rangle+\left\langle v^{\prime}, \nabla(v)\right\rangle\right] \otimes \xi=0 .
\end{aligned}
$$

Therefore $\nabla^{\ell}(\epsilon)=0 \in L^{\infty}$, for all $\ell$.

Once we have established that $\epsilon \in W^{\infty, \infty}\left(M ; \operatorname{Hom}\left(E \otimes E^{\prime} \otimes F ; F\right)\right)$, the second part of the proposition follows from Proposition 2.5.

Although we shall not use the following result in this paper, it is an interesting general result that may allow us to compare Sobolev norms with their Euclidean counter-parts.

Let $\mathcal{U}=\left(U_{i}\right)_{i \in I}$ be a covering of $M$, that is, $M=\cup_{i \in I} U_{i}$. Recall that its covering multiplicity is $N(\mathcal{U})=N\left(\left(U_{i}\right)_{i \in I}\right)$

(20) $N(\mathcal{U}):=\max \left\{r \mid \exists i_{1}, i_{2}, \ldots, i_{r} \in I\right.$ distinct with $\left.U_{i_{1}} \cap U_{i_{2}} \cap \ldots \cap U_{i_{r}} \neq \emptyset\right\}$.

Also, recall that $\mathcal{U}=\left(U_{i}\right)_{i \in I}$ of $M$ is uniformly locally finite if $N(\mathcal{U})<\infty$. Assume that the index set $I$ labeling the open sets of the covering $\mathcal{U}$ is countable. Then we also let

$$
\|\| u \|_{\mathcal{U}, s, p}:= \begin{cases}\left(\sum_{j \leq s, i \in I}\left\|\nabla^{i} u\right\|_{L^{p}\left(U_{i}\right)}^{p}\right)^{1 / p} & \text { if } 1 \leq p<\infty \\ \sup _{j \leq s, i \in I}\left\|\nabla^{i} u\right\|_{L^{\infty}\left(U_{i}\right)} & \text { if } p=\infty\end{cases}
$$

Proposition 2.11. We have \|\|$u\left\|_{\mathcal{U}_{, s, \infty}}=\right\| u \|_{W^{s, \infty}(M)}$. If $1 \leq p<\infty$, then $\|u\|_{W^{s, \infty}(M)} \leq\|\| u\left\|_{\mathcal{U}_{, s, p}} \leq N(\mathcal{U})^{1 / p}\right\| u \|_{W^{s, \infty}(M)}$.

Proof. This follows from the definitions of the norms $\|\cdot\|_{W^{s, \infty}(M)}$ and \|\|$\cdot\|\|_{\mathcal{U}, s, p}$ and, for $f \geq 0$ measurable, the inequalities

$$
\int_{M} f \mathrm{dvol} \leq \sum_{i \in I} \int_{U_{i}} f \mathrm{dvol} \leq N(\mathcal{U}) \int_{M} f \mathrm{dvol} .
$$

This completes the proof.

To continue our study of $\nabla$-Sobolev spaces, we need to take a look also at " $\nabla$ differential operators," which we introduce in the next section. 
2.5. Global, geometric $\nabla$-differential operators. We now start our study of differential operators. We shall consider globally defined differential operators on $M$ with smooth coefficients acting on sections of smooth vector bundles. We provide several definitions. We are especially interested in definitions that do not rely on local coordinates (unlike the classical one). We may assume that these vector bundles are endowed with metrics and metric preserving connections, denoted generically by $\nabla$, as agreed above. We let $\nabla^{0}=i d$.

As mentioned already, all our vector bundles will be smooth. Also, we shall consider differential operators with smooth coefficients, unless otherwise mentioned. The case of differential operators with non-smooth coefficients is to a large extent very similar.

Recall that $V^{\otimes k}:=V \otimes V \otimes \ldots \otimes V$ (k-times) and that $V^{\otimes 0}:=\mathbb{C}$. It will be convenient to consider the "truncated Fock space"

$$
\mathcal{F}_{\mu}^{M}(E):=\oplus_{j=0}^{\mu} T^{* \otimes j} M \otimes E .
$$

We endow the truncated Fock space $\mathcal{F}_{m}^{M}(E)$ with the induced connection from $E$ and $T^{*} M$. Given $\left.a \in \mathcal{C}^{\infty}\left(M ; \mathcal{F}_{\mu}^{M}(E) ; F\right)\right)$, we shall write $a^{[j]} \in \mathcal{C}^{\infty}\left(M ; \operatorname{Hom}\left(T^{* \otimes j} M \otimes\right.\right.$ $E ; F)), j=0, \ldots, \mu$, for the resulting component and we shall also define $a \cdot \nabla^{\text {tot }}:=$ $\sum_{j=0}^{\mu} a^{[j]} \nabla^{j}$.

Definition 2.12. Let $E, F \rightarrow M$ be vector bundles, with $E$ endowed with a connection, and let $a=\left(a^{[0]}, a^{[1]}, \ldots, a^{[\mu]}\right)$ be a suitable section of $\left.\operatorname{Hom}\left(\mathcal{F}_{\mu}^{M}(E) ; F\right)\right)$. A $\nabla$-differential operator is a map

$$
P=a \cdot \nabla^{t o t}:=\sum_{j=0}^{\mu} a^{[j]} \nabla^{j}: \mathcal{C}_{\mathrm{c}}^{\infty}(M ; E) \rightarrow \mathcal{C}_{\mathrm{c}}^{\infty}(M ; F) .
$$

We let $\operatorname{ord}(P)$ denote the least $\mu$ for which such a writing exists and call it the order of $P$. Suitable extensions by continuity of $P$ will also be called $\nabla$-differential operators and will be denoted by the same letter.

Unless stated otherwise, all our differential operators will have smooth coefficients. Thus, by "a $\nabla$-differential operator" will mean a " $\nabla$-differential operator with smooth coefficients." The case of operators with non-smooth coefficients will only rarely be considered, but it usually can be treated in a similar way.

Notations 2.13. Let us introduce now some further notation and terminology related a differential operator $P:=a \cdot \nabla^{t o t}:=\sum_{j=0}^{\mu} a^{[j]} \nabla^{j}$ as in the definition.

- If $a \in \mathcal{C}^{\infty}\left(M ; \operatorname{Hom}\left(\mathcal{F}_{\mu}^{M}(E) ; F\right)\right)$, we shall say that $P$ has $\mathcal{C}^{\infty}$-coefficients. The set of such operators is denoted $\operatorname{Diff}_{\nabla}^{\mu}(M ; E, F)$.

Assume now also that $E$ and $F$ are endowed with hermitian metrics.

- If $a \in W_{\nabla}^{\ell, \infty}\left(M ; \operatorname{Hom}\left(\mathcal{F}_{\mu}^{M}(E) ; F\right)\right)$, we shall say that $P$ has coefficients in $W^{\ell, \infty}$.

- If, in fact, $\ell=0$, we shall say that $P$ has bounded coefficients.

- On the other hand, if $\ell=\infty$, we shall say that $P$ has totally bounded (or $\left.\mathcal{C}_{b}^{\infty}\right)$ coefficients. The set of such operators is denoted $\operatorname{Diff}_{b, \nabla}^{\mu}(M ; E, F)$.

We shall often drop the index $\nabla$ from $\operatorname{Diff}_{\nabla}^{\mu}(M ; E, F)$ and $\operatorname{Diff}_{b, \nabla}^{\mu}(M ; E, F)$. Here are some comments.

Remark 2.14. We use the notation of Definition 2.12. 
(1) If $j \geq 2$, the coefficient $a^{[j]}$ in $\left.a=\left(a^{[0]}, a^{[1]}, \ldots, a^{[\mu]}\right) \in \mathcal{C}^{\infty}\left(M ; \mathcal{F}_{\mu}^{M}(E) ; F\right)\right)$ is not uniquely determined by the map $P=a \cdot \nabla^{\text {tot }}:=\sum_{j=0}^{\mu} a^{[j]} \nabla^{j}$ : $\mathcal{C}_{\mathrm{c}}^{\infty}(M ; E) \rightarrow \mathcal{C}_{\mathrm{c}}^{\infty}(M ; F)$.

(2) Peetre's Theorem $[34,48]$ characterizes the explicit structure of $\nabla$-differential operators and shows that such an operator is, indeed, a classical differential operator in any coordinate chart.

(3) Note that in our approach avoiding local coordinates, one also needs to consider vector valued Sobolev spaces (with values in tensor products of the cotangent bundle) even if one is interested only in scalar equations.

We have the following "easy" continuities.

Lemma 2.15. The definition of $\nabla$-differential operators gives for $k \in \mathbb{N}$ :

(1) $\nabla: W_{\nabla}^{k+1, p}(M ; E) \rightarrow W_{\nabla}^{k, p}\left(M ; T^{*} M \otimes E\right)$ is continuous.

(2) For $P$ as in Definition 2.12 with coefficients in $W^{\ell, \infty}$, we have that

$$
P=\sum_{j=0}^{\mu} a_{j} \nabla^{j}: W_{\nabla}^{k+\mu, p}(M ; E) \rightarrow W_{\nabla}^{k, p}(M ; E), \quad 0 \leq k \leq \ell,
$$

is well-defined and continuous.

Proof. For all $j \leq k$ and $u \in W_{\nabla}^{k+1, p}(M ; E)$, we have $\nabla^{j} \nabla u \in L^{2}\left(T^{\otimes}(j+1) M \otimes E\right)$, by definition, and hence $\nabla u \in W_{\nabla}^{k, p}(M ; E)$, again by definition. The continuity in the second part follows by combining the first part with Proposition 2.5, which gives the continuity of the maps

$$
W_{\nabla}^{k, \infty}\left(M ; \operatorname{Hom}\left(T M^{* \otimes j} \otimes E ; F\right)\right) \otimes W_{\nabla}^{k, p}\left(M ; T^{*} M^{\otimes j} \otimes E\right) \rightarrow W_{\nabla}^{k, p}(M ; F) .
$$

In particular, $P$ is well-defined with the stated domain and range.

We want to extend this mapping property to other spaces. It extends to $k \geq 0$ real immediately by interpolation, since our fractional order Sobolev spaces were defined by interpolation, see, for instance, [41, 52], or [54, Chapter 2]. To extend also to $k \leq 0$, we shall need also the following basic algebraic properties, whose statements rely on the following notation.

Notations 2.16. 2.13 Recall that $\operatorname{Diff}^{\mu}(M ; E, F)$ is the set of $\nabla$-differential operators $\mathcal{C}_{c}^{\infty}(M ; E) \rightarrow \mathcal{C}_{c}^{\infty}(M ; F)$ of order $\leq \mu$ with smooth coefficients. (On the rare occasions when we shall need to show the dependence on the connection, we shall also write $\operatorname{Diff}_{\nabla}^{\mu}(M ; E, F)$ for this space). Similarly, $\operatorname{Diff}_{b}^{\mu}(M ; E, F) \subset \operatorname{Diff}^{\mu}(M ; E, F)$ is the set of $\nabla$-differential operators of order $\leq \mu$ with $\mathcal{C}_{b}^{\infty}$-coefficients. We shall also write:

- $\operatorname{Diff}^{\mu}(M ; E):=\operatorname{Diff}^{\mu}(M ; E, E)$ and $\operatorname{Diff}_{b}^{\mu}(M ; E):=\operatorname{Diff}_{b}^{\mu}(M ; E, E)$;

- $\operatorname{Diff}^{\infty}(M ; E)=\cup_{\mu} \operatorname{Diff}^{\mu}(M ; E)$ and $\operatorname{Diff}_{b}^{\infty}(M ; E)=\cup_{\mu} \operatorname{Diff}_{b}^{\mu}(M ; E)$, which will be seen to be algebras in the next proposition.

- We will omit $E$ from the notation when $E=F=\mathbb{C}$. Thus $\operatorname{Diff}^{\mu}(M):=$ $\operatorname{Diff}^{\mu}(M ; \mathbb{C})=\operatorname{Diff}^{\mu}(M ; \mathbb{C}, \mathbb{C})$ and $\operatorname{Diff}_{b}^{\mu}(M):=\operatorname{Diff}_{b}^{\mu}(M ; \mathbb{C})=\operatorname{Diff}_{b}^{\mu}(M ; \mathbb{C}, \mathbb{C})$, and so on.

Proposition 2.17. We use the notation introduced in Definition 2.12 and 2.13.

(i) The spaces $\operatorname{Diff}^{\mu}(M ; E, F)$ and $\operatorname{Diff}_{b}^{\mu}(M ; E, F)$ are linear vector spaces.

(ii) Let $P \in \operatorname{Diff}^{j}(M ; E, F)$ and $Q \in \operatorname{Diff}^{N}(M ; F, G)$, then $Q P \in \operatorname{Diff}^{N+j}(M ; E, G)$. 
(iii) If $P$ and $Q$ have $\mathcal{C}_{b}^{\infty}$-coefficients, then $Q P$ has $\mathcal{C}_{b}^{\infty}$-coefficients as well.

(iv) In particular,

$$
\operatorname{Diff}^{\infty}(M ; E):=\bigcup_{\mu} \operatorname{Diff}^{\mu}(M ; E, E) \text { and } \operatorname{Diff}_{b}^{\infty}(M ; E):=\bigcup_{\mu} \operatorname{Diff}_{b}^{\mu}(M ; E, E)
$$

are algebras.

Proof. The statement (i) follows right away from the definitions of the spaces $\operatorname{Diff}_{b}^{\mu}(M ; E, F) \subset \operatorname{Diff}^{\mu}(M ; E, F)$ and the fact that the spaces of $\mathcal{C}_{\mathrm{b}}^{\infty} \subset \mathcal{C}^{\infty}$-sections (over $M$ of various vector bundles) are vector spaces.

By linearity (part (i)), it is enough to assume that $Q=b \nabla^{j}$ and $P=a \nabla^{N}$, where $a$ and $b$ are suitable sections of endomorphism bundles, as in Definition 2.12. We shall prove the statements (ii) and (iii) by induction on $j$.

Let us prove the statement (ii). If $j=0$, this is true since $Q P=b a \nabla^{N}$ and $b a$ is a smooth endomorphism. Moreover, Remark 2.1 gives

$$
\left(b \nabla^{j}\right) \circ\left(a \nabla^{N}\right)(u)=b \nabla^{j-1}\left[(\nabla a) \nabla^{N} u+(1 \otimes a) \nabla^{N+1} u\right],
$$

so the first statement follows by induction on $j$, since $(\nabla a) \nabla^{N}+(1 \otimes a) \nabla^{N+1}$ is also a $\nabla$-differential operator with smooth coefficients (if $a \in \mathcal{C}^{\infty}$, then $\nabla(a)$ and $1 \otimes a$ are also in $\left.\mathcal{C}^{\infty}\right)$.

For the statement (iii), let us assume that $P$ and $Q$ have $\mathcal{C}_{\mathrm{b}}^{\infty}$-coefficients and prove that $Q P$ has the same property. We then proceed in exactly the same way, using the formula (24). So assume $a, b \in \mathcal{C}_{\mathrm{b}}^{\infty}$. When $j=0$, we obtain similarly that $Q P=b a \nabla^{N}$ and $b a$ is in $\mathcal{C}_{\mathrm{b}}^{\infty}$ due to Proposition 2.5. For $j>0$, we notice that $(\nabla a) \nabla^{N}+(1 \otimes a) \nabla^{N+1}$ has $\mathcal{C}_{\mathrm{b}}^{\infty}$ coefficients (if $a \in \mathcal{C}_{\mathrm{b}}^{\infty}$, then $\nabla(a)$ and $1 \otimes a$ are also in $\mathcal{C}_{\mathrm{b}}^{\infty}$ ), which yields the induction step.

The statement (iv) follows right away from the previous two.

We can now address the dependence of the definitions of $\nabla$-Sobolev spaces and $\nabla$-differential operators on $\nabla$.

Remark 2.18. The composition property proved in Proposition 2.17(ii) gives that, in particular, if we are given two connections, $\widetilde{\nabla}$ and $\nabla$ on $E$ (so that $\widetilde{\nabla}-\nabla=: A \in$ $\left.\mathcal{C}^{\infty}(M ; \operatorname{End}(E))\right)$, then $\operatorname{Diff}_{\nabla}^{\mu}(M ; E, F)=\operatorname{Diff}_{\tilde{\nabla}}^{\mu}(M ; E, F)$, which justifies dropping the index $\nabla$. The analogous equality $\operatorname{Diff}_{b, \nabla}^{\mu}(M ; E, F)=\operatorname{Diff}_{b, \widetilde{\nabla}}^{\mu}(M ; E, F)$ for operators with $\mathcal{C}_{\mathrm{b}}^{\infty}$-coefficients is true if, and only if, $A \in \mathcal{C}_{\mathrm{b}}^{\infty}(M ; \operatorname{End}(E))$. This is stated as part of the following proposition.

We can now prove the independence on the connection $\nabla$ of our definitions, under certain conditions.

Proposition 2.19. Let $A \in \mathcal{C}^{\infty}(M ; \operatorname{End}(E)), A^{*}=-A$, and $\widetilde{\nabla}=\nabla+A$. Let also $p \in[1, \infty]$ and $\mu \in \mathbb{N}$. We then have the following:

(i) $A \in W_{\nabla}^{\infty, \infty}(M ; \operatorname{End}(E))$ if, and only if, $\operatorname{Diff}_{b, \nabla}^{\mu}(M ; E, F)=\operatorname{Diff}_{b, \widetilde{\nabla}}^{\mu}(M ; E, F)$.

(ii) If $A \in W_{\nabla}^{\ell-1, \infty}(M ; \operatorname{End}(E))$, then there is $C_{\ell} \geq 1$ that depends only on $\|A\|_{W_{\nabla}^{\ell-1, \infty}(M)}$ such that, for all $u \in \mathcal{C}_{c}^{\infty}(M ; E)$,

$$
C_{\ell}^{-1}\|u\|_{W_{\nabla}^{\ell, p}(M)} \leq\|u\|_{W_{\vec{\nabla}}^{\ell, p}(M)} \leq C_{\ell}\|u\|_{W_{\nabla}^{\ell, p}(M)} .
$$

Consequently,

$$
W_{\nabla}^{\ell, p}(M ; E)=W_{\widetilde{\nabla}}^{\ell, p}(M ; E) .
$$


Proof. The first statement follows from Proposition 2.17. Indeed, the third statement of this proposition combined with the assumption that $A \in C_{b}^{\infty}(M ; \operatorname{End}(E))$ shows that the operator $(\nabla+A)^{j}$ has $\mathcal{C}_{\mathrm{b}}^{\infty}$-coefficients, for all $0 \leq j \leq \mu$. Moreover, any operator $P$ in $\operatorname{Diff}_{b, \widetilde{\nabla}}^{\mu}(M ; E, F)$ has the form $P=\sum_{j=0}^{\mu} \widetilde{a}^{[j]} \widetilde{\nabla}^{j}$, where $\widetilde{a}^{[j]}$ are $\mathcal{C}_{\mathrm{b}}^{\infty}$-coefficients. Thus, $P$ can be written in the equivalent form $P=$ $\sum_{j=0}^{\mu} \widetilde{a}^{[j]}(\nabla+A)^{j}=\sum_{j=0}^{\mu} b^{[j]} \nabla^{j}$, where $b^{[j]}$ are $\mathcal{C}_{\mathrm{b}}^{\infty}$-coefficients as well. This shows the inclusion $\operatorname{Diff}_{b, \widetilde{\nabla}}^{\mu}(M ; E, F) \subseteq \operatorname{Diff}_{b, \nabla}^{\mu}(M ; E, F)$. The converse inclusion follows by symmetry.

Next we assume that $\operatorname{Diff}_{b, \nabla}^{\mu}(M ; E, F)=\operatorname{Diff}_{b, \widetilde{\nabla}}^{\mu}(M ; E, F)$. Then the operators $\nabla$ and $\widetilde{\nabla}=\nabla+A$ belong to $\operatorname{Diff}_{b, \nabla}^{\mu}(M ; E, F)$, and, thus, $A=\widetilde{\nabla}-\nabla \in$ $C_{b}^{\infty}(M ; \operatorname{End}(E))$, since the later is a vector space.

The second statement is proved by induction on $\ell$ using Propositions 2.5 and 2.17. Indeed, first of all, if $\ell=0$ then $W_{\nabla}^{0, p}(M ; E)=L^{p}(M ; E)=W_{\widetilde{\nabla}}^{0, p}(M ; E)$ and the property follows, with $C_{0}=1$.

Assume now that the property we want to prove holds for $\ell-1 \geq 0$ and show it for $\ell$. The definition of the norm in $W_{\nabla}^{\ell, p}(M ; E)$ and the induction hypothesis imply that

$$
\begin{aligned}
\|u\|_{W_{\nabla}^{\ell, p}(M)}^{p} & \leq\|u\|_{W_{\nabla}^{\ell-1, p}(M)}^{p}+\|\nabla u\|_{W_{\nabla}^{\ell-1, p}(M)}^{p} \\
& =\|u\|_{W_{\nabla}^{\ell-1, p}(M)}^{p}+\|\widetilde{\nabla} u-A u\|_{W_{\nabla}^{\ell-1, p}(M)}^{p} \\
& \leq C_{\ell-1}^{p}\|u\|_{W_{\nabla}^{\ell-1, p}(M)}^{p}+2^{p-1}\|\widetilde{\nabla} u\|_{W_{\nabla}^{\ell-1, p}(M)}^{p}+2^{p-1}\|A u\|_{W_{\nabla}^{\ell-1, p}(M)}^{p} \\
& \leq C_{\ell-1}^{p}\left(1+2^{p-1}\right)\|u\|_{W_{\vec{\nabla}}^{\ell, p}(M)}^{p}+2^{p-1} C_{\ell-1, p}^{p}\|A\|_{W_{\nabla}^{\ell-1, \infty}(M)}^{p}\|u\|_{W_{\nabla}^{\ell-1, p}(M)}^{p} \\
& \leq C_{\ell-1}^{p} 2^{p-1}\left[2+C_{\ell-1, p}^{p}\|A\|_{W_{\nabla}^{\ell-1, \infty}(M)}^{p}\right]\|u\|_{W_{\nabla}^{\ell, p}(M)}^{p},
\end{aligned}
$$

where $C_{\ell-1, p}$ is the (absolute) constant of Proposition 2.5. The first desired inequality for the norm then follows if $C_{\ell-1}^{p} 2^{p-1}\left[2+C_{\ell-1, p}^{p}\|A\|_{W_{\nabla}^{\ell-1, \infty}(M)}^{p}\right]=: C_{\ell}^{p}$. Since we can bound $\|A\|_{W_{\vec{\nabla}}^{\ell-1, \infty}(M)}^{p}$ in terms of $\|A\|_{W_{\nabla}^{\ell-1, \infty}(M)}^{p}$, by the induction hypothesis, the second of the desired inequalities for the norms follows by symmetry. In particular, we deduce the equality $W_{\nabla}^{\ell, p}(M ; E)=W_{\widetilde{\nabla}}^{\ell, p}(M ; E)$.

\section{Mixed DiffEREnTial operators AND totally BOUNDED VeCtor FIELDS}

In this section we look at a different type of differential operators, which we call "mixed differential operators," since they will be used to relate the $\nabla$-differential operators of the previous section to the classical differential operators. To study them, we introduce the Fréchet finiteness condition (FFC) and we show that if (FFC) is satisfied, then the mixed differential operators coincide with the $\nabla$ ones.

3.1. The Fréchet finite generation condition. It is known [43, p. 71] that every vector bundle $E \rightarrow M$ on a manifold has an embedding $\Phi: E \rightarrow M \times \mathbb{R}^{N}$ into a trivial bundle. In this subsection, we shall use the existence of such an embedding for $E=T M$ and we shall deduce some geometric consequences.

Remark 3.1. Let $\Phi: T M \rightarrow M \times \mathbb{R}^{N}$ be a smooth embedding of the tangent bundle into a trivial vector bundle. We endow the trivial vector bundle with the constant 
metric. Then the transpose $\Phi^{\top}: M \times \mathbb{R}^{N} \rightarrow T M$ is onto. (The transpose is the analog of the adjoint, but in the real case.) Moreover, $\Phi^{\top} \Phi$ is a smooth, invertible section of $\operatorname{End}(T M)$. Let $\Psi:=\left(\Phi^{\top} \Phi\right)^{-1} \Phi^{\top}$, so that $\Psi \in \mathcal{C}^{\infty}\left(M ; \operatorname{Hom}\left(\mathbb{R}^{N} ; T M\right)\right.$ and $\Psi \Phi=1$. Let

$$
Z_{1}:=\Psi\left(e_{1}\right), Z_{2}:=\Psi\left(e_{2}\right), \ldots, Z_{N}:=\Psi\left(e_{N}\right) \in \mathcal{C}^{\infty}(M ; T M)
$$

be the vector fields corresponding to the constant basis $\left(e_{j}\right)_{j=1}^{N}$ of $\mathbb{R}^{N}$ via $\Phi^{\top}$. Since $\Psi \in \mathcal{C}^{\infty}\left(M ; \operatorname{Hom}\left(\mathbb{R}^{N} ; T M\right)\right)$, we have that $Z_{j}$ are all in $\mathcal{C}^{\infty}(M ; T M)$. Let

$$
\xi_{j}:=p_{j} \circ \Phi: T M \rightarrow \mathbb{R}
$$

be the 1 -form obtained from the projection of $\mathbb{R}^{N}$ onto the $j$ th component. Then $\xi_{j} \in \mathcal{C}^{\infty}\left(M ; T^{*} M\right)$ and the relation $\Psi \Phi=1$ gives, for every $X \in \mathcal{C}^{\infty}(M ; T M)$,

$$
X=\Psi(\Phi(X))=\Psi\left(\sum_{j=1}^{N} \xi_{j}(X) e_{j}\right)=\sum_{j=1}^{N} \xi_{j}(X) Z_{j}
$$

In particular,

$$
\mathcal{C}^{\infty}(M ; T M)=\sum_{j=1}^{N} \mathcal{C}^{\infty}(M) Z_{j}
$$

Let $\omega \in \mathcal{C}_{\mathrm{b}}^{\infty}\left(M ; T^{*} M\right)$. By evaluating $\omega$ in the above relation, we obtain that $\omega(X)=\sum_{j=1}^{N} \xi_{j}(X) \omega\left(Z_{j}\right)$, and hence we have the dual relation $\omega=\sum_{j=1}^{N} \omega\left(Z_{j}\right) \xi_{j}$.

We now take a look at a different global definition of differential operators.

Definition 3.2. We let $\widetilde{\operatorname{Diff}}^{\mu}(M ; E, F)$ to be the set of all linear operators of order $\leq \mu$ linearly generated by $a \nabla_{X_{1}} \ldots \nabla_{X_{r}}, 0 \leq r \leq \mu, a \in \mathcal{C}^{\infty}(M ; \operatorname{Hom}(E, F)), X_{j} \in$ $\mathcal{C}^{\infty}(M ; T M)$. An operator $P$ of this type will be called a mixed differential operator of order $\leq \mu$. If $a \in \mathcal{C}_{\mathrm{b}}^{\infty}(M ; \operatorname{Hom}(E, F))$ and all $X_{j} \in \mathcal{W}_{b}(M):=\mathcal{C}_{\mathrm{b}}^{\infty}(M ; T M)$, then $P$ is called a a mixed differential operator of order $\leq \mu$ with $\mathcal{C}_{b}^{\infty}$-coefficients, and the set of all such operators is denoted by $\widetilde{\operatorname{Diff}}_{b}^{\mu}(M ; E, F)$.

As we will see in Subsection 3.3, mixed differential operators are, sometimes, easier to deal with than $\nabla$-differential operators and form a convenient intermediate class between $\nabla$ - and classical differential operators. The following lemma is standard, except for the fact that the system $\left(Z_{j}\right)$ is only a system of generators of $\mathcal{C}^{\infty}(M ; T M)$ as a $\mathcal{C}^{\infty}(M)$-module, and not a basis.

Lemma 3.3. Let $Z_{j}$ and $\xi_{j}$ be as in Remark 3.1.

(i) If $X \in \mathcal{C}^{\infty}(M ; T M)$, then $\nabla_{X} \in \operatorname{Diff}^{1}\left(M ; E, T^{*} M \otimes E\right)$.

(ii) $\nabla^{E}: \mathcal{C}_{c}^{\infty}(M ; E) \rightarrow \mathcal{C}_{c}^{\infty}\left(M ; T^{*} M \otimes E\right)$ satisfies $\nabla^{E}=\sum_{j=1}^{N} \xi_{j} \otimes \nabla_{Z_{j}}^{E}$.

(iii) For any $X \in \mathcal{C}^{\infty}(M ; T M)$, we have $\operatorname{div}(X) \in \mathcal{C}^{\infty}(M)$.

(iv) We have $\nabla_{Z_{i}} Z_{j}=\sum_{k} G_{i j}^{k} Z_{k}$, where $G_{i j}^{k} \in \mathcal{C}^{\infty}(M)$.

(v) We have $\left[Z_{i}, Z_{j}\right]=\sum_{k} L_{i j}^{k} Z_{k}$, where $L_{i j}^{k} \in \mathcal{C}^{\infty}(M)$.

Proof. Let $Z_{1}, Z_{2}, \ldots, Z_{N} \in \mathcal{C}^{\infty}(M ; T M)$ be the vector fields introduced in Remark 3.1 .

To prove (i), let $i_{X}: T^{*} M \otimes E \rightarrow E$ be the contraction with the vector $X \in$ $\mathcal{C}^{\infty}(M ; T M)$. Then $i_{X} \in \mathcal{C}^{\infty}\left(M ; \operatorname{Hom}\left(T^{*} M \otimes E ; E\right)\right)$ since $X \in \mathcal{C}^{\infty}(M ; T M)$, and hence $\nabla_{X}=i_{X} \circ \nabla$ is a $\nabla$-differential operator with smooth coefficients, by definition. 
To prove (ii), let $u \in \mathcal{C}_{\mathrm{c}}^{\infty}(M ; E)$ and $X \in \mathcal{C}_{\mathrm{c}}^{\infty}(M ; T M)$ be arbitrary. Let $i_{X}$ : $T^{*} M \otimes E \rightarrow E$ be the contraction with the vector $X \in \mathcal{C}_{\mathrm{c}}^{\infty}(M ; T M), i_{X}(u)=\langle X, u\rangle$. Then the formula $X=\sum_{j=1}^{N} \xi_{j}(X) Z_{j}$ of Remark 3.1 gives

$$
\left\langle X, \nabla^{E}(u)\right\rangle=\nabla_{X}^{E}(u)=\sum_{j=1}^{N} \xi_{j}(X) \nabla_{Z_{j}}^{E}(u)=\left\langle X, \sum_{j=1}^{N} \xi_{j} \otimes \nabla_{Z_{j}}^{E}(u)\right\rangle .
$$

Since $u \in \mathcal{C}_{\mathrm{c}}^{\infty}\left(M ; T^{*} M\right)$ and $X \in \mathcal{C}_{\mathrm{c}}^{\infty}(M ; T M)$ were arbitrary, the result follows.

To prove (iii), let $X_{1}, X_{2}, \ldots, X_{n}$ be a local orthonormal basis of $T M$. Then we obtain

$$
\begin{aligned}
\operatorname{div}(X) & =\operatorname{tr}(\nabla X) \\
& =\sum_{i=1}^{n}\left(\nabla_{X_{i}} X, X_{i}\right) \\
& =\sum_{j=1, k, l}^{n} \xi_{k}\left(X_{j}\right) \xi_{l}\left(X_{j}\right)\left(\nabla_{Z_{k}} X, Z_{l}\right) \\
& =\sum_{j=1, k, l}^{n}\left(\xi_{k}, \xi_{l}\right)\left(\nabla_{Z_{k}} X, Z_{l}\right)
\end{aligned}
$$

since the map $T^{*} M \ni \xi \rightarrow\left(\left(\xi, X_{j}\right)\right)_{j=1}^{n}$ is an isometry (since $\left(X_{i}\right)$ was chosen to be an orthonormal basis). Hence, the result follows.

To prove (iv), we notice that, since $\nabla_{Z_{i}} Z_{j} \in \mathcal{C}^{\infty}(M ; T M)$, we have $\nabla_{Z_{i}} Z_{j}=$ $\sum_{k=1}^{N} \xi_{k}\left(\nabla_{Z_{i}} Z_{j}\right) Z_{k}$, by Remark 3.1, and hence we can take $G_{i j}^{k}:=\xi_{k}\left(\nabla_{Z_{i}} Z_{j}\right)$, which is in $\mathcal{C}_{\mathrm{b}}^{\infty}(M)$ by Proposition 2.5. For (v), we proceed in exactly the same way since the Lie bracket of vector fields $\left[Z_{i}, Z_{j}\right] \in \mathcal{C}^{\infty}(M ; T M)$.

We have the following generation property for mixed differential operators.

Proposition 3.4. Let $\mu, \nu \in \mathbb{N}$.

(i) $\widetilde{\operatorname{Diff}}^{\nu}(M ; F, G) \widetilde{\operatorname{Diff}}^{\mu}(M ; E, F) \subset \widetilde{\operatorname{Diff}}^{\mu+\nu}(M ; E, G)$.

(ii) $\widetilde{\operatorname{Diff}}^{\mu}(M ; E, F)=\operatorname{Diff}^{\mu}(M ; E, F)$.

(iii) Let $Z_{1}, Z_{2}, \ldots, Z_{N} \in \mathcal{C}^{\infty}(M)$ be a systems of generators for $\mathcal{C}^{\infty}(M)$ as in 3.1, then $\operatorname{Diff}^{\mu}(M ; E, F)$ is linearly generated by $a \nabla_{X_{1}} \nabla_{X_{2}} \ldots \nabla_{X_{r}}$, where $r \leq \mu$, $X_{1}, X_{2}, \ldots, X_{r} \in\left\{Z_{1}, Z_{2}, \ldots, Z_{N}\right\}$ and $a \in \mathcal{C}^{\infty}(M ; \operatorname{Hom}(E, F))$.

Proof. We prove the first statement by induction on $\mu$. For $\mu=0$, the property (i) follows from the multiplication properties

$$
\mathcal{C}^{\infty}(M ; \operatorname{Hom}(F ; G)) \mathcal{C}^{\infty}(M ; \operatorname{Hom}(E ; F)) \subset \mathcal{C}^{\infty}(M ; \operatorname{Hom}(E ; G)) .
$$

The induction step is obtained using also the equation $\nabla_{X} a=\nabla_{X}(a)+a \nabla_{X}$.

To prove (ii), let us notice that Lemma 3.3(i) states that $\nabla_{X} \in \operatorname{Diff}^{1}(M ; E, F)$. The composition property of Proposition 2.17(ii), yields the inclusion $\widetilde{\operatorname{Diff}}^{\mu}(M ; E, F) \subset$ $\operatorname{Diff}^{\mu}(M ; E, F)$. Let us prove now the opposite inclusion.

Let $\tau_{\xi}(\zeta):=\xi \otimes \zeta$ and $\nabla=\sum_{i=1}^{N} \tau_{\xi_{i}} \nabla_{Z_{i}}$, as in Lemma 3.3. This gives $\nabla \in$ $\widetilde{\operatorname{Diff}}^{1}\left(M ; E, T^{*} M \otimes E\right)$. Part (i), already proved, then proves, by induction on $j$, that $a \nabla^{j} \in \widetilde{\operatorname{Diff}}^{\mu}(M ; E, F)$ if $a \in \mathcal{C}^{\infty}(M ; \operatorname{Hom}(E ; F))$ and $j \leq \mu$. Therefore $\operatorname{Diff}^{\mu}(M ; E, F) \subset \widetilde{\operatorname{Diff}}^{\mu}(M ; E, F)$. Hence we have equality. 
Let $\mathcal{D}_{\mu}$ be the linear span of $a \nabla_{X_{1}} \nabla_{X_{2}} \ldots \nabla_{X_{r}}$, where $r \leq \mu$ and $X_{1}, X_{2}, \ldots, X_{r} \in$ $\left\{Z_{1}, Z_{2}, \ldots, Z_{N}\right\}$ and $a \in \mathcal{C}^{\infty}(M ; \operatorname{Hom}(E, F))$. It is enough to prove that $\mathcal{D}_{\mu}=$ $\operatorname{Diff}^{\mu}(M ; E, F)$.

Let $Q:=a \nabla_{X_{1}} \nabla_{X_{2}} \ldots \nabla_{X_{r}}$, where $r \leq \mu$ and $X_{1}, X_{2}, \ldots, X_{r} \in \mathcal{C}^{\infty}(M)$ and $a \in \mathcal{C}^{\infty}(M ; \operatorname{Hom}(E, F))$. We shall prove, by induction on $r$, that $Q \in \mathcal{D}_{\mu}$, that is, that $Q$ is a linear combination of terms of the same kind, but with all $X_{j} \in$ $\left\{Z_{1}, Z_{2}, \ldots, Z_{N}\right\}$. By induction, we can assume that this is true for products of up to $r-1$ covariant derivatives. The induction step is obtained using the equation $\nabla_{X} a=\nabla_{X}(a)+a \nabla_{X}$. Indeed, let us consider then $a$ and $b$ be $\mathcal{C}_{\mathrm{b}}^{\infty}$-endomorphisms, $X_{2}, X_{3}, \ldots, X_{r} \in\left\{Z_{1}, Z_{2}, \ldots, Z_{N}\right\}$ and $X_{1}$ an arbitrary smooth vector field. Then

$$
Q_{1}:=a \nabla_{X_{1}} b \nabla_{X_{2}} \ldots \nabla_{X_{r}}=a\left[X_{1}(b)+b \sum_{j=1}^{N} \xi_{j}\left(X_{1}\right) \nabla_{Z_{j}}\right] \nabla_{X_{2}} \ldots \nabla_{X_{r}}
$$

We thus have $Q_{1} \in \mathcal{D}_{\mu}$. This proves the equality of $\mathcal{D}_{\mu}$ and $\widetilde{\operatorname{Diff}}^{\mu}(M ; E, F)$.

We obtain the following consequence.

Corollary 3.5. Let $\left(Z_{j}\right), 1 \leq j \leq N, Z_{j} \in \mathcal{C}^{\infty}(M ; T M)$ be as in Remark 3.1. Then $\operatorname{Diff}^{\mu}(M ; E, F)$ is linearly generated by $a \nabla_{Z_{k_{1}}}^{E} \ldots \nabla_{Z_{k_{r}}}^{E}$, where $1 \leq k_{1} \leq k_{2} \leq$ $\cdots \leq k_{r} \leq N, r \leq \mu$, and $a \in \mathcal{C}^{\infty}(M ; \operatorname{Hom}(E ; F))$.

Proof. We know that $\operatorname{Diff}^{\mu}(M ; E, F)$ is linearly generated by terms of the form $a \nabla_{Z_{k_{1}}}^{E} \ldots \nabla_{Z_{k_{r}}}^{E}$, where $k_{1}, k_{2}, \ldots, k_{r} \in\{1,2, \ldots, N\}, r \leq \mu, a \in \mathcal{C}^{\infty}(M ; \operatorname{Hom}(E ; F))$. It just remains to show that we can choose the indices $k$ to form a non-decreasing sequence. To this end, we shall use the relation

$$
R^{E}(X, Y):=\left[\nabla_{X}^{E}, \nabla_{Y}^{E}\right]-\nabla_{[X, Y]}^{E} \in \mathcal{C}^{\infty}(M ; \operatorname{End}(E)) .
$$

This shows, that, up to lower order terms, we can commute the operators $\nabla_{Z_{j}}^{E}$. The proof is completed by induction.

3.2. Totally bounded vector fields and the (FFC) condition. Recall that we write $\operatorname{Diff}_{b}^{\infty}(M):=\operatorname{Diff}_{b}^{\infty}(M ; \mathbb{C})$ for the algebras of differential operators on $M$ with $\mathcal{C}_{\mathrm{b}}^{\infty}(M)$-coefficients introduced in Proposition 2.17(iv) when $E$ is the trivial vector bundle with fiber $\mathbb{C}$. Then, by sepparating the order zero part of a differential operator, we obtain

$$
\operatorname{Diff}_{b}^{1}(M)=\mathcal{C}_{\mathrm{b}}^{\infty}(M) \oplus \mathcal{W}_{b}(M)
$$

where

$$
\mathcal{W}_{b}(M):=\mathcal{C}_{\mathrm{b}}^{\infty}(M ; T M):=W^{\infty, \infty}(M ; T M)
$$

that is, the space of bounded vector fields on $M$ all of whose covariant derivatives are bounded. Then $\mathcal{W}_{b}(M)$ is a module over $\mathcal{C}_{\mathrm{b}}^{\infty}(M)$ with respect to multiplication by Proposition 2.5. This space (which will turn out to be a Lie algebra) will play an important role in what follows and this section is devoted, to a large extent, to the study of their role in the definition of $\nabla$-Sobolev spaces and $\nabla$-differential operators.

Lemma 3.6. Let $\mathcal{W}_{b}(M):=\mathcal{C}_{b}^{\infty}(M ; T M)$

(i) If $X \in \mathcal{W}_{b}(M)$, then $\nabla_{X} \in \operatorname{Diff}_{b}^{1}\left(M ; E, T^{*} M \otimes E\right)$.

(ii) $\mathcal{W}_{b}(M)$ is a Lie algebra, that is, $[X, Y]:=X Y-Y X \in \mathcal{W}_{b}(M)$ for all $X, Y \in \mathcal{W}_{b}(M)$.

(iii) If $X, Y \in \mathcal{W}_{b}(M)$, then $\nabla_{X} Y \in \mathcal{W}_{b}(M)$. 
Proof. To prove (i), let $i_{X}: T^{*} M \otimes E \rightarrow E$ be the contraction with the vector $X \in \mathcal{W}_{b}(M)$. Then $i_{X} \in W^{\infty, \infty}\left(M ; \operatorname{Hom}\left(T^{*} M \otimes E ; E\right)\right)$ since $X \in \mathcal{W}_{b}(M)$, and hence $\nabla_{X}=i_{X} \circ \nabla$ is a $\nabla$-differential operator with coefficients in $\mathcal{C}_{\mathrm{b}}^{\infty}$, by definition.

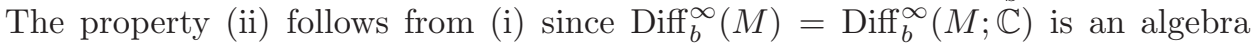
(see Proposition 2.17(iv)). Property (iii) follows from (i) and the "easy" mapping property of Lemma 2.15 (which gives, in particular, that $P \in \operatorname{Diff}_{b}^{\mu}(M ; E, F)$ maps $W_{\nabla}^{\infty, \infty}(M ; E)$ to $W_{\nabla}^{\infty, \infty}(M ; F)$ continuously.

Definition 3.7. We say that $(M, g)$ satisfies the Fréchet finiteness condition (FFC) if, there exists $N \in \mathbb{N}$ and an isometric (vector bundle) embedding $\Phi: T M \subset M \times$ $\mathbb{R}^{N}, \Phi \in W^{\infty, \infty}\left(M ; \operatorname{Hom}\left(T M ; \mathbb{R}^{N}\right)\right)$, where on $\mathbb{R}^{N}$ we consider the flat connection.

This condition may seem strong, but it was proved in Lemma 3.1 of [30] that it is satisfied by a manifold with bounded geometry. In fact, in that paper, instead of constructing a $\Phi$ with the property that it is an isometry, it was proved that $\Phi^{-1} \in \mathcal{C}_{\mathrm{b}}^{\infty}\left(M ; \operatorname{Hom}\left(\mathbb{R}^{N} ; T M\right)\right)$. By replacing that $\Phi$ with its polar part, one can assume it to be isometric. In particular, the (FFC) property is hereditary, in the sense that if it is satisfied by a manifold $M$, then it is satisfied by any open subset $M_{0} \subset M$. Indeed, it is enough to restrict $\Phi$ to $M_{0}$.

We have the following analog of Remark 3.1.

Remark 3.8. We use the notation of Remark 3.1. Since we have assumed that $\Phi$ is an isometry now, we have $\Psi=\Phi^{\top}: M \times \mathbb{R}^{N} \rightarrow T M$ and, for all $1 \leq j \leq N$, we have

$$
\begin{gathered}
Z_{j}:=\Phi^{\top}\left(e_{j}\right) \in \mathcal{W}_{b}(M):=\mathcal{C}_{\mathrm{b}}^{\infty}(M ; T M) \text { and } \\
\xi_{j}:=p_{j} \circ \Phi \in \mathcal{C}_{\mathrm{b}}^{\infty}\left(M ; T^{*} M\right)
\end{gathered}
$$

so that $\mathcal{W}_{b}(M)=\sum_{j=1}^{N} \mathcal{C}_{\mathrm{b}}^{\infty}(M) Z_{j}$. The relations

$$
X=\sum_{j=1}^{N} \xi_{j}(X) Z_{j} \text { and } \omega=\sum_{j=1}^{N} \omega\left(Z_{j}\right) \xi_{j}
$$

remain, of course, valid. The set $\left\{Z_{1}, Z_{2}, \ldots, Z_{N}\right\}$ will be called a Fréchet system of generators for $\mathcal{W}_{b}(M)$.

Let div $:=-d^{\prime}$ be the negative of the dual map $d^{\prime}: \mathcal{C}_{\mathrm{c}}^{\infty}(M ; T M) \rightarrow \mathcal{C}_{\mathrm{c}}^{\infty}(M)$ of $d: \mathcal{C}_{\mathrm{c}}^{\infty}(M) \rightarrow \mathcal{C}_{\mathrm{c}}^{\infty}\left(M ; T^{*} M\right)$. We shall need the following result.

Lemma 3.9. Assume $M$ satisfies the (FFC) condition and let $\xi_{j} \in \mathcal{C}_{b}^{\infty}\left(M ; T^{*} M\right)$ and $Z_{j} \in \mathcal{W}_{b}(M):=\mathcal{C}_{b}^{\infty}(M ; T M), j=1, \ldots, N$, be as in Remark 3.8.

(i) If $X \in \mathcal{W}_{b}(M)$, then $\nabla_{X} \in \operatorname{Diff}_{b}^{1}\left(M ; E, T^{*} M \otimes E\right)$.

(ii) For any $X \in \mathcal{W}_{b}(M)$, we have $\operatorname{div}(X) \in \mathcal{C}_{b}^{\infty}(M)$.

(iii) We have $\nabla_{Z_{i}} Z_{j}=\sum_{k} G_{i j}^{k} Z_{k}$, where $G_{i j}^{k} \in W^{\infty, \infty}(M)$.

(iv) We have $\left[Z_{i}, Z_{j}\right]=\sum_{k} L_{i j}^{k} Z_{k}$, where $L_{i j}^{k} \in W^{\infty, \infty}(M)$.

Proof. To prove (i), let $u \in \mathcal{C}_{\mathrm{c}}^{\infty}(M ; E)$ and $X \in \mathcal{C}_{\mathrm{b}}^{\infty}(M ; T M)$ be arbitrary. Let $i_{X}: T^{*} M \otimes E \rightarrow E$ be the contraction with the vector $X \in \mathcal{W}_{b}(M), i_{X}(u)=\langle X, u\rangle$. Then the second displayed formula in the last remark gives

$$
\left\langle X, \nabla^{E}(u)\right\rangle=\nabla_{X}^{E}(u)=\sum_{j=1}^{N} \xi_{j}(X) \nabla_{Z_{j}}^{E}(u)=\left\langle X, \sum_{j=1}^{N} \xi_{j} \otimes \nabla_{Z_{j}}^{E}(u)\right\rangle .
$$

Since $u \in \mathcal{C}_{\mathrm{c}}^{\infty}(M ; E)$ and $X \in \mathcal{C}_{\mathrm{b}}^{\infty}(M ; T M)$ were arbitrary, the result follows. 
Let $X_{1}, X_{2}, \ldots, X_{n}$ be a local orthonormal basis of $T M$. Let $Z_{1}, Z_{2}, \ldots, Z_{N} \in$ $\mathcal{W}_{b}(M):=W^{\infty, \infty}(M ; T M)$ be the Fréchet system of generating vector fields introduced in Remark 3.8. Then we obtain

$$
\begin{aligned}
\operatorname{div}(X) & =\sum_{i=1}^{n}\left(\nabla_{X_{i}} X, X_{i}\right) \\
& =\sum_{j=1, k, l}^{n} \xi_{k}\left(X_{j}\right) \xi_{l}\left(X_{j}\right)\left(\nabla_{Z_{k}} X, Z_{l}\right) \\
& =\sum_{j=1, k, l}^{n}\left(\xi_{k}, \xi_{l}\right)\left(\nabla_{Z_{k}} X, Z_{l}\right)
\end{aligned}
$$

by the isometry property of the map $T^{*} M \ni \xi \rightarrow\left(\left(\xi, X_{j}\right)\right)_{j=1}^{n}\left(\right.$ since $\left(X_{i}\right)$ was chosen to be an orthonormal basis).

Lemma 3.6 gives that $\nabla_{Z_{i}} Z_{j} \in \mathcal{W}_{b}(M)$. Hence $\nabla_{Z_{i}} Z_{j}=\sum_{k=1}^{N} \xi_{k}\left(\nabla_{Z_{i}} Z_{j}\right) Z_{k}$ and we can take $G_{i j}^{k}:=\xi_{k}\left(\nabla_{Z_{i}} Z_{j}\right)$, which is in $\mathcal{C}_{\mathrm{b}}^{\infty}(M)$ by Proposition 2.5. For (iv), we proceed in exactly the same way by using Lemma 3.6(iii).

We can now formulate and prove the following proposition, which provides us with the usual properties of the Hilbert space adjoints $\nabla_{X}^{*}$ and $\nabla^{*}$.

Proposition 3.10. Let $\mu \in \mathbb{N}$ and $1<p<+\infty$ and let us assume that $M$ satisfies the Fréchet finiteness condition (Definition 3.7). Then we also have the following properties.

(i) If $X \in \mathcal{W}_{b}(M)$, then $\nabla_{X}^{*}=-\nabla_{X}-\operatorname{div}(X) \in \operatorname{Diff}_{b}^{1}\left(M ; T^{*} M \otimes E, E\right)$.

(ii) $\nabla^{*} \in \operatorname{Diff}_{b}^{1}\left(M ; T^{*} M \otimes E, E\right)$.

Proof. To prove (i), let $\operatorname{div}=-d^{\prime}: \mathcal{C}_{\mathrm{c}}^{\infty}\left(M ; T^{*} M\right) \rightarrow \mathcal{C}_{\mathrm{c}}^{\infty}(M)$, the negative of the transpose of the de Rham differential. Then $\operatorname{div}(f X)=f \operatorname{div}(X)+X(f)$. Let us write $\nabla_{X}$ for $\nabla_{X}^{E}$. Then, using formula (1), we obtain for all $\xi, \eta \in \mathcal{C}_{\mathrm{c}}^{\infty}(M ; E)$

$$
\begin{aligned}
\left(\nabla_{X} \xi, \eta\right)_{E} & =X(\xi, \eta)_{E}-\left(\xi, \nabla_{X} \eta\right)_{E} \\
& =\operatorname{div}\left((\xi, \eta)_{E} X\right)-(\xi, \eta)_{E} \operatorname{div}(X)-\left(\xi, \nabla_{X} \eta\right)_{E}
\end{aligned}
$$

and then, integrating over $\mathrm{M}$ and using the assumption that $\xi$ and $\eta$ have compact support (so the integral of the "div" part is zero), we get

$$
\int_{M}\left(\nabla_{X} \xi, \eta\right)_{E} \text { dvol }=-\int_{M}\left(\xi, \operatorname{div}(X) \eta+\nabla_{X} \eta\right)_{E} \text { dvol } .
$$

Thus, $\nabla_{X}^{*}=-\nabla_{X}-\operatorname{div}(X)$, as stated, and hence $\nabla_{X}^{*}$ belongs to $\operatorname{Diff}_{b}^{1}\left(M ; T^{*} M \otimes\right.$ $E, E)$ by Lemma 3.6(i) and by Lemma 3.9(ii).

Let $\tau_{\xi}(\zeta):=\xi \otimes \zeta$. Then, using the notation of Remarks 3.1 and 3.8, we can reformulate the result of Lemma 3.9 (ii) as

$$
\nabla=\sum_{i=1}^{N} \tau_{\xi_{i}} \nabla_{Z_{i}}
$$

The relation (ii) thus follows from this relation by taking adjoints and using (i) and the composition property of Proposition 2.17(ii).

We can now prove the following extension of the standard continuity of differential operators. 
Corollary 3.11. Let $1<p<\infty$. If $P=\sum_{j=0}^{\mu} a_{j} \nabla^{j}$ and $a_{j} \in \mathcal{C}_{b}^{\infty}(M ; \operatorname{Hom}(E ; F))$, that is, if $P$ is a $\nabla$-differential operator with coefficients in $\mathcal{C}_{b}^{\infty}$, then $P$ extends by continuity to maps

$$
\begin{gathered}
W_{0, \nabla}^{s, p}(M ; E) \rightarrow W_{0, \nabla}^{s-\mu, p}(M ; F), \quad s \in \mathbb{N}, s \geq \mu, \\
W_{\nabla}^{s, p}(M ; E) \rightarrow W_{\nabla}^{s-\mu, p}(M ; F), \quad s \in \mathbb{R} .
\end{gathered}
$$

Proof. We have already seen that $P: W_{\nabla}^{s, p}(M ; E) \rightarrow W_{\nabla}^{s-\mu, p}(M ; F)$ is continuous if $s \geq \mu, s \in \mathbb{N}$, see Lemma 2.15(2). Moreover, a differential operator will send compactly supported sections to compactly supported sections. The first statement thus follows.

Let us turn now to the general case. Since for non-integer $s$, the spaces $W_{\nabla}^{s, p}$ are defined by interpolation between consecutive integers, it suffices to prove our statement for integer values of $s$. (The general case is obtained by interpolation.) Furthermore, using Proposition 2.17, we see that it is also enough to consider the case $P=\nabla$ (so $\mu=1)$. We have then two possibilities for $s$, either $s \geq \mu=1$ or $s \leq 0$. The first case was already proved, as we have just mentioned. To prove the case $s \leq 0$, recall that the adjoint operator is also a $\nabla-$ differential operator by Proposition 3.10 and that the negative order Sobolev spaces $W^{-s, p}\left(M ; E^{*}\right):=W_{0, \nabla}^{s, p^{\prime}}(M ; E)^{*}$, see Equation (6). As the statement is known for $\nabla^{*}: W_{0, \nabla}^{1-s, p^{\prime}}(M ; E)^{*} \rightarrow W_{0, \nabla}^{-s, p^{\prime}}(M ; E)^{*}$ since $\nabla^{*} \in \operatorname{Diff}_{b}^{1}\left(M ; T^{*} M \otimes E, E\right)$ by the first part (since we reduced to $s \in \mathbb{Z}_{+}$), the desired statement is obtained by taking adjoints.

We shall use $P$ to denote all the maps in Corollary 3.11 induced by the original $P$. We shall not need the case $s \notin \mathbb{Z}$, but that case can usually be handled by interpolation [41, 54].

Recall that $M$ is an $n$-dimensional manifold with metric $g$ and $\nabla^{L C}$ is the associated Levi-Civita connection.

Corollary 3.12. Let $1<p<\infty$. Let $\rho, f_{0}: M \rightarrow(0, \infty)$ be admissible weights with respect to the metric $g_{0}=\rho^{-2} g$. Let $P=\sum_{j=0}^{\mu} a_{j} \nabla^{j}$ and $a_{j} \in \mathcal{C}_{b}^{\infty}\left(M, g_{0} ; \operatorname{Hom}(E ; F)\right)$, that is $P$ is a $\nabla$-differential operator with coefficients in $\mathcal{C}_{b}^{\infty}$ with respect to the metric $g_{0}$. Then $P$ extends by continuity to maps

$$
f_{0} W_{\nabla, \rho}^{\ell, p}(M ; E) \rightarrow f_{0} \rho^{-\mu} W_{\nabla, \rho}^{\ell-\mu, p}(M ; F), \quad \ell \in \mathbb{N} .
$$

Proof. Recall that the relation between the weighted Sobolev space $f_{0} W_{\nabla^{L C}, \rho}^{\ell, p}(M ; E)$ (defined with respect to the metric $g$ ) and the classical Sobolev space $W^{\ell, p}\left(M, g_{0} ; E\right.$ ) (defined with respect to the metric $g_{0}=\rho^{-2} g$ ) is given by the formula

$$
f_{0} W_{\nabla, \rho}^{\ell, p}(M ; E)=f_{0} \rho^{-\frac{n}{p}} W^{\ell, p}\left(M, g_{0} ; E\right) .
$$

In addition, the assumption that $f_{0}$ is admissible shows that $f_{0}^{-1} \rho^{\mu} P f_{0}$ is a $\nabla$ differential operator with coefficients in $\mathcal{C}_{\mathrm{b}}^{\infty}$ with respect to the metric $g_{0}$, that is, $f_{0}^{-1} P f_{0}$ and $P$ are both $\nabla$-differential operators of the same type. Then, in view of Corollary 3.11, this operator extends by continuity to the maps

$$
W^{\ell, p}\left(M, g_{0} ; E\right) \rightarrow W^{\ell-\mu, p}\left(M, g_{0} ; F\right), \quad \ell \in \mathbb{N} .
$$

Then formula (28) and the mapping property (29) show that $P$ extends by continuity to the maps given in (27). 
3.3. Bounded mixed differential operators. The (FFC) condition gives the following finite generation property for the algebra of $\nabla$-differential operators with $\mathcal{C}_{\mathrm{b}}^{\infty}$-coefficients. It is analogous to Proposition 3.4.

Proposition 3.13. Let $\mu \in \mathbb{N}$. Assume $M$ satisfies (FFC) and let $Z_{1}, Z_{2}, \ldots, Z_{N} \in$ $\mathcal{C}^{\infty}(M)$ be a Fréchet systems of generators for $\mathcal{C}^{\infty}(M)$ as in 3.1,

(i) $\widetilde{\operatorname{Diff}}_{b}^{\nu}(M ; F, G) \widetilde{\operatorname{Diff}}_{b}^{\mu}(M ; E, F) \subset \widetilde{\operatorname{Diff}}_{b}^{\mu+\nu}(M ; E, G)$.

(ii) $\widetilde{\operatorname{Diff}}_{b}^{\mu}(M ; E, F)=\operatorname{Diff}_{b}^{\mu}(M ; E, F)$.

(iii) then $\operatorname{Diff}_{b}^{\mu}(M ; E, F)$ is linearly generated by $a \nabla_{X_{1}} \nabla_{X_{2}} \ldots \nabla_{X_{r}}$, where $r \leq \mu$, $X_{1}, X_{2}, \ldots, X_{r} \in\left\{Z_{1}, Z_{2}, \ldots, Z_{N}\right\}$ and $a \in \mathcal{C}_{b}^{\infty}(M ; \operatorname{Hom}(E, F))$.

Proof. The proof is completely similar to that of Proposition 3.4, by using the composition property

$$
\mathcal{C}_{\mathrm{b}}^{\infty}(M ; \operatorname{Hom}(F ; G)) \mathcal{C}_{\mathrm{b}}^{\infty}(M ; \operatorname{Hom}(E ; F)) \subset \mathcal{C}_{\mathrm{b}}^{\infty}(M ; \operatorname{Hom}(E ; G)),
$$

and the properties $\nabla_{X} \in \operatorname{Diff}_{b}^{1}(M ; E, F)$ if $X \in \mathcal{W}_{b}(M):=\mathcal{C}_{\mathrm{b}}^{\infty}(M ; T M), \nabla \in$ $\widetilde{\operatorname{Diff}}_{m}^{1}\left(M ; E, T^{*} M \otimes E\right)$, and $\nabla_{X} a=\nabla_{X}(a)+a \nabla_{X} \in \mathcal{C}_{\mathrm{b}}^{\infty}(M ; \operatorname{Hom}(E, F))$ if $a \in$ $\mathcal{C}_{\mathrm{b}}^{\infty}(M ; \operatorname{Hom}(E, F))$ and $X \in \mathcal{W}_{b}(M)$ instead of the corresponding statements in that proof.

Let us recall that a vector bundle $E \rightarrow M$ is said to have totally bounded curvature if its curvature $R^{E} \in \mathcal{C}_{\mathrm{b}}^{\infty}\left(M ; \Lambda^{2} T^{*} M \otimes \operatorname{End}(E)\right)$. Recall that if $M$ satisfies the (FFC) condition, then $\widetilde{\operatorname{Diff}^{\mu}}=\operatorname{Diff}^{\mu}$ and $\widetilde{\operatorname{Diff}}_{b}^{\mu}=\operatorname{Diff}_{b}^{\mu}$. We next show that we can choose the vector fields $X_{j}$ in the above proposition in the right order.

Corollary 3.14. Assume $M$ satisfies the (FFC) condition and let $\left(Z_{j}\right), 1 \leq j \leq N$, be a Fréchet generating system for $\mathcal{W}_{b}(M)$. Let us assume also that $E \rightarrow M$ has totally bounded curvature

(i) If $X, Y \in \mathcal{W}_{b}(M)$, then $\nabla_{X}^{E} \nabla_{Y}^{E}-\nabla_{Y}^{E} \nabla_{X}^{E}-\nabla_{[X, Y]}^{E} \in \mathcal{C}_{b}^{\infty}(M ; \operatorname{End}(E))$.

(ii) Consequently, $\operatorname{Diff}_{b}^{\mu}(M ; E, F)$ is linearly generated by $a \nabla_{Z_{k_{1}}}^{E} \ldots \nabla_{Z_{k_{r}}}^{E}$, where $1 \leq k_{1} \leq k_{2} \leq \ldots \leq k_{r} \leq N, r \leq \mu$, and $a \in \mathcal{C}_{b}^{\infty}(M ; \operatorname{Hom}(E ; F))$.

Proof. The statement (i) follows also from the formula (25) taking into account that, in this case, $R^{E}(X, Y) \in \mathcal{C}_{\mathrm{b}}^{\infty}(M ; \operatorname{End}(E))$, since $R^{E} \in \mathcal{C}_{\mathrm{b}}^{\infty}\left(M ; \Lambda^{2} T^{*} M \otimes \operatorname{End}(E)\right)$ and $X, Y \in \mathcal{W}_{b}(M)$.

Finally, the last part is proved in the same way Corollary 3.5, but taking into account also (i).

3.4. Equivalent definitions of $\nabla$-Sobolev spaces. Let $Z_{1}, Z_{2}, \ldots, Z_{N} \in \mathcal{W}_{b}(M):=$ $W^{\infty, \infty}(M ; T M)$ be a Fréchet system of generators of $\mathcal{W}_{b}(M)$ as $\mathcal{C}_{\mathrm{b}}^{\infty}$-module, and let $\left\{\xi_{1}, \xi_{2}, \ldots, \xi_{\mu}\right\}$ be the dual system, as in Remark 3.8. We shall write $\mathbf{k}=$ $\left(k_{1}, k_{2}, \ldots, k_{\mu}\right), 1 \leq k_{1}, k_{2}, \ldots k_{\mu} \leq N$.

Lemma 3.15. Assume that $M$ satisfies the (FFC) condition. Then any $w \in$ $L^{p}\left(M ; T^{* \otimes \mu} M \otimes E\right)$ can be written as

$$
w=\sum_{\mathbf{k}} \xi_{k_{1}} \otimes \xi_{k_{2}} \otimes \ldots \otimes \xi_{k_{\mu}} \otimes i_{Z_{\mu}} i_{Z_{\mu-1}} \ldots i_{Z_{1}}(w) .
$$

Proof. The result follows from the relation $\omega(X)=\sum_{j=1}^{N} \xi_{j}(X) \omega\left(Z_{j}\right)$ (see Remark 3.1), the definition of $i_{Z_{j}}$, which shows that $\omega\left(Z_{j}\right)=\left\langle\omega, Z_{j}\right\rangle=i_{Z_{j}}(\omega)$, and the 
expression $\left(\xi_{i} \otimes \xi_{j}\right)(X \otimes Y)=\xi_{i}(X) \xi_{j}(Y)$ of the tensor product of two 1-forms $\xi_{i}(X)$ and $\xi_{j}(Y)$.

The following result gives several alternative description of Sobolev spaces $W_{\nabla}^{s, p}(M ; E)$, $s \in \mathbb{N}, 1 \leq p \leq \infty$, in terms of vector fields similar to [30, Proposition 3.2], where part of this result was proved for manifolds with bounded geometry. Let us record the following easy lemma.

Lemma 3.16. Assume that $M$ satisfies the (FFC) condition. Then $w \in L^{p}\left(M ; T^{* \otimes \mu} M \otimes\right.$ $E)$ if, and only if, aw $\in L^{p}(M ; E)$ for all $a \in \mathcal{C}_{b}^{\infty}\left(M ; \operatorname{Hom}\left(T^{* \otimes \mu} M \otimes E ; E\right)\right)$.

Proof. If $w \in L^{p}\left(M ; T^{* \otimes \mu} M \otimes E\right)$ and $a \in \mathcal{C}_{\mathrm{b}}^{\infty}\left(M ; \operatorname{Hom}\left(T^{* \otimes \mu} M \otimes E ; E\right)\right)$, then we have already seen that $a w \in L^{p}(M ; E)$.

Conversely, let $Z_{1}, Z_{2}, \ldots, Z_{N} \in \mathcal{W}_{b}(M):=W^{\infty, \infty}(M ; T M)$ be a Fréchet system of generators of $\mathcal{W}_{b}(M)$ as $\mathcal{C}_{\mathrm{b}}^{\infty}$-module, as in Remark 3.8. Let $\left\{\xi_{1}, \xi_{2}, \ldots, \xi_{\mu}\right\}$ be the dual system, again as in that remark. Let $\mathbf{k}=\left(k_{1}, k_{2}, \ldots, k_{\mu}\right), 1 \leq k_{1}, k_{2}, \ldots k_{\mu} \leq$ $N$. We let $a$ range through the composition of contractions $i_{Z_{\mu}} i_{Z_{\mu-1}} \ldots i_{Z_{1}}$, which recovers $a$. Then Lemma 3.15 implies that

$$
w=\sum_{\mathbf{k}} \xi_{k_{1}} \otimes \xi_{k_{2}} \otimes \ldots \otimes \xi_{k_{\mu}} \otimes i_{Z_{\mu}} i_{Z_{\mu-1}} \ldots i_{Z_{1}}(w) .
$$

If all $a w \in L^{p}(M ; E)$, then by taking $a:=\xi_{k_{1}} \otimes \xi_{k_{2}} \otimes \ldots \otimes \xi_{k_{\mu}} \otimes i_{Z_{\mu}} i_{Z_{\mu-1}} \ldots i_{Z_{1}}(w)$, we get that all

$$
\xi_{k_{1}} \otimes \xi_{k_{2}} \otimes \ldots \otimes \xi_{k_{\mu}} \otimes i_{Z_{\mu}} i_{Z_{\mu-1}} \ldots i_{Z_{1}}(w) \in L^{p}\left(M ; T^{* \otimes \mu} M \otimes E\right),
$$

and hence $w \in L^{p}\left(M ; T^{* \otimes \mu} M \otimes E\right)$.

The following type of descriptions is often used in the setting of weighted Sobolev spaces, see, for instance [12, 16, 21, 23, 24, 39, 40, 46].

Proposition 3.17. Let $Z_{1}, Z_{2}, \ldots, Z_{N} \in \mathcal{W}_{b}(M):=W^{\infty, \infty}(M ; T M)$ be a Fréchet system of generators of $\mathcal{W}_{b}(M)$ as $\mathcal{C}_{b}^{\infty}$-module, as in Remark 3.8, if $M$ satisfies the (FCC) condition. Let $s \in \mathbb{N}$ and $1 \leq p \leq \infty$. Then the following spaces all coincide with $W_{\nabla}^{s, p}(M ; E)$ under the listed additional conditions:

(i) $W_{\nabla}^{s, p}(M ; E)=\left\{u \mid P u \in L^{p}(M ; E), \forall P \in \operatorname{Diff}_{b}^{s}(M ; E, F)\right\}$.

(ii) $W_{\nabla}^{s, p}(M ; E)=\left\{u \mid P u \in L^{p}(M ; E), \forall P \in \operatorname{Diff}_{b}^{s}(M ; E)\right\}$, provided that $M$ satisfies the $(F F C)$ condition.

(iii) $W_{\nabla}^{\ell, p}(M ; E)=\left\{u \mid \nabla_{Z_{k_{1}}}^{E} \nabla_{Z_{k_{2}}}^{E} \ldots \nabla_{Z_{k_{j}}}^{E} u \in L^{p}(M ; E), j \leq \ell, 1 \leq k_{1}, k_{2}, \ldots, k_{j} \leq\right.$ $N\}$, provided that $M$ satisfies the (FFC) condition.

(iv) $W_{\nabla}^{\ell, p}(M ; E)=\left\{u \mid \nabla_{Z_{k_{1}}}^{E} \nabla_{Z_{k_{2}}}^{E} \ldots \nabla_{Z_{k_{j}}}^{E} u \in L^{p}(M ; E), j \leq \ell, 1 \leq k_{1} \leq k_{2} \leq\right.$ $\left.\ldots \leq k_{j} \leq N\right\}$ provided that $M$ satisfies the $(F F C)$ condition and $E$ has totally bounded curvature.

Proof. The first characterization of the Sobolev spaces $W_{\nabla}^{\mu, p}(M ; E)$ follows from the definition of $\nabla$-Sobolev spaces (Definition 2.2) and $\nabla$-differential operators (Definition 2.12), since it is enough to take $P$ among the monomials $\nabla^{j}, 0 \leq$ $j \leq \mu$. (Indeed, any $P \in \operatorname{Diff}_{b}^{s}(M ; E, F)$ has the form $P=\sum_{j=0}^{\mu} a_{j} \nabla^{j}$ with $a_{j} \in \mathcal{C}_{\mathrm{b}}^{\infty}(M ; \operatorname{Hom}(E ; F))$.)

The second point is similar. Indeed, we have $w \in L^{p}\left(M ; T^{* \otimes k} M \otimes E\right.$ if, and only if, $a w \in L^{p}\left(M ; T^{* \otimes k} M \otimes E\right.$ for all $a \in \mathcal{C}_{\mathrm{b}}^{\infty}\left(M ; \operatorname{Hom}\left(T^{* \otimes k} M \otimes E\right)\right.$. By applying 
this observation to $w:=\nabla^{j} u, j \leq \mu$, and using Lemma 3.16, the definitions of $\nabla$-Sobolev spaces and $\nabla$-differential operators, we obtain the result.

The third and fourth points are also similar. They follow by combining (i) with Lemma 3.16 and Proposition ?? (for (iii)), respectively Corollary 3.14 for (iv).

3.5. Bidifferential operators and Dirichlet forms. Let $M_{1}$ and $M_{2}$ be two topological spaces and let

$$
\pi_{j}: M_{1} \times M_{2} \rightarrow M_{j}
$$

be the projection onto the $j$ th component, $j=1,2$. For any two real or complex vector bundles $E_{j} \rightarrow M_{j}$, we let

$$
E_{1} \otimes E_{2}:=\pi_{1}^{*}\left(E_{1}\right) \otimes \pi_{2}^{*}\left(E_{2}\right) \rightarrow M_{1} \times M_{2}
$$

be the external tensor product of $E_{1}$ and $E_{2}$. It is a vector bundle on $M_{1} \times M_{2}$. More concretely, if $x_{j} \in M_{j}$ and $E_{j, x_{j}}$ is the fiber of $E_{j}$ above $x_{j}$, then the fiber of $E_{1} \otimes E_{2}$ above $\left(x_{1}, x_{2}\right)$ is $E_{1, x_{1}} \otimes E_{2, x_{2}}$. If $M_{1}=M_{2}=M$, in which case we shall always regard $M$ as being diagonally embedded in $M \times M$, then, of course,

$$
\left.E_{1} \otimes E_{2}\right|_{M}=E_{1} \otimes E_{2} .
$$

Remark 3.18. If $M_{j}$ are smooth manifolds and $E_{j} \rightarrow M_{j}$ are smooth vector bundles endowed with connections, $j=1,2$, then $E_{1} \otimes E_{2}$ is endowed with the canonically induced connection from $\pi_{j}^{*}\left(E_{j}\right)$ (which acts trivially on the fiber $M_{3-j}$ of $\pi_{j}$ : $\left.M_{1} \times M_{2} \rightarrow M_{j}\right)$. Let us take a closer look at this induced connection on $E_{1} \otimes E_{2}$. We first notice that we have a canonical isomorphism

$$
T\left(M_{1} \times M_{2}\right) \simeq T M_{1} \times T M_{2} \simeq \pi_{1}^{*}\left(T M_{1}\right) \oplus \pi_{2}^{*}\left(T M_{2}\right) .
$$

Let $p_{j}: T^{*}\left(M_{1} \times M_{2}\right) \rightarrow \pi_{j}^{*}\left(T^{*} M_{j}\right)$ be the induced projections and let

$$
\nabla^{E_{1} \otimes E_{2}}=\nabla_{1}+\nabla_{2} \text { where } \nabla_{j}:=\left(p_{j} \otimes i d_{E_{1} \otimes E_{2}}\right) \circ \nabla^{E_{1} \otimes E_{2}} .
$$

If $u_{j} \in \mathcal{C}_{\mathrm{c}}^{\infty}\left(M_{j} ; E_{j}\right), j=1,2$, we let $v:=u_{1} \otimes u_{2} \in \mathcal{C}_{\mathrm{c}}^{\infty}\left(M_{1} \times M_{2} ; E_{1} \otimes E_{2}\right)$, that is, $v\left(x_{1}, x_{2}\right):=\left(u_{1} \otimes u_{2}\right)\left(x_{1}, x_{2}\right):=u\left(x_{1}\right) \otimes u_{2}\left(x_{2}\right)$. We then obtain

$$
\nabla_{1} v=\nabla u_{1} \otimes u_{2}, \nabla_{2} v=u_{1} \otimes \nabla u_{2}, \text { and hence } \nabla_{1} \nabla_{2}=\nabla_{2} \nabla_{1} \text {. }
$$

Let $V$ be a complex vector space or vector bundle and let $\bar{V}$ denote the conjugate space to $V$. More precisely, $\bar{V}=V$ as additive groups, but with the external multiplication $\bar{z} \bar{v}=\overline{z v}, z \in \mathbb{C}, v \in V$, where $\bar{v}$ denotes the image in $\bar{V}$ of an element $v \in V$.

Definition 3.19. Let $E, F \rightarrow M$ be two smooth vector bundles endowed with connections. A $\nabla$-bidifferential operator on $(E, F)$ is a linear map

$$
\mathfrak{b}^{\nabla}: \mathcal{C}_{\mathrm{c}}^{\infty}(M \times M ; E \otimes \bar{F}) \rightarrow \mathcal{C}_{\mathrm{c}}^{\infty}(M)
$$

of the form $\mathfrak{b}^{\nabla} v=\left.P v\right|_{M}$, where $P: \mathcal{C}_{\mathrm{c}}^{\infty}(M \times M ; E \otimes \bar{F}) \rightarrow \mathcal{C}_{\mathrm{c}}^{\infty}(M \times M)$ is a $\nabla$-differential operator with smooth coefficients.

If we can choose $P$ to have $\mathcal{C}_{\mathrm{b}}^{\infty}$-coefficients (that is in $\operatorname{Diff}_{b}(M \times M ; E \otimes \bar{F}, \mathbb{C})$ ), then we say that $\mathfrak{b}^{\nabla}$ has $\mathcal{C}_{\mathrm{b}}^{\infty}$-coefficients as well.

Bidifferential operators appeared also in the framework of deformation quantization. Let us obtain a more explicit form of the $\nabla$-bidifferential operators. 
Remark 3.20. We use the notation of Definition 3.19 and let $\pi_{j}: M \times M \rightarrow M, j=$ 1,2 , be the two projections. Let $\nabla^{E \otimes \bar{F}}=\nabla_{1}+\nabla_{2}$ be the decomposition of Remark 3.18. Thus, if $u \in \mathcal{C}_{\mathrm{c}}^{\infty}(M ; E), w \in \mathcal{C}_{\mathrm{c}}^{\infty}(M ; F)$, and $v:=u \otimes \bar{w} \in \mathcal{C}_{\mathrm{c}}^{\infty}(M \times M ; E \otimes \bar{F})$, then $\nabla_{1} v=\nabla u \otimes \bar{w}, \nabla_{2} v=u \otimes \nabla \bar{w}$. Therefore

$$
\begin{aligned}
P v:=a \cdot \nabla^{t o t} v=\sum_{j=0}^{\mu} a^{[j]} \nabla^{j} v & =\sum_{i+j \leq \mu} \tilde{\mathfrak{a}}_{i j}\left(\nabla_{1}^{i} \nabla_{2}^{j} v\right) \\
& =\sum_{i+j \leq \mu} \tilde{\mathfrak{a}}_{i j}\left[\left(\nabla^{i} u\right) \otimes\left(\nabla^{j} \bar{w}\right)\right] \in \mathcal{C}_{\mathrm{c}}^{\infty}(M \times M),
\end{aligned}
$$

where each $\tilde{\mathfrak{a}}_{i j} \in \mathcal{C}^{\infty}\left(M \times M ;\left[\left(T^{* \otimes i} M \otimes E\right) \otimes\left(T^{* \otimes j} M \otimes \bar{F}\right)\right]^{\prime}\right)$ is obtained in a canonical (linear) way from $a \in \mathcal{C}^{\infty}\left(M \times M ; \mathcal{F}_{\mu}^{M \times M}(E \otimes \bar{F})^{\prime}\right)$. In particular, if $\tilde{a} \in \mathcal{C}_{\mathrm{b}}^{\infty}$, then all $\tilde{a}_{i j} \in \mathcal{C}_{\mathrm{b}}^{\infty}$ as well and their bounds are controlled by the bounds for $a$. Let us assume also that $F$ is endowed with a Hermitian metric $(,)_{F}$, regarded

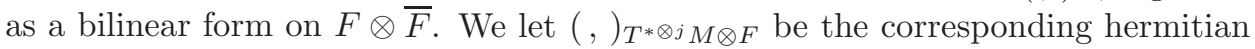
form (i.e. bilinear form on $T^{* \otimes j} M \otimes F \otimes T^{* \otimes j} M \otimes \bar{F}$ ). Then there exist unique

$$
\mathfrak{a}_{i j} \in \mathcal{C}^{\infty}\left(M ; \operatorname{Hom}\left(T^{* \otimes i} M \otimes E ; T^{* \otimes j} M \otimes F\right)\right)
$$

such that $\left(\mathfrak{a}_{i j} \xi, \eta\right)=\left.\tilde{a}_{i j}\right|_{M}(\xi \otimes \bar{\eta})$ and hence we have the following canonical form for $\mathfrak{b}^{\nabla}$ :

$$
\mathfrak{b}^{\nabla} v(x)=\mathfrak{b}^{\nabla}(u \otimes \bar{w})(x)=\sum_{i+j \leq \mu}\left(\mathfrak{a}_{i j}(x) \nabla^{i} u(x), \nabla^{j} w(x)\right)_{T^{* \otimes j} M \otimes F} .
$$

If $\mathfrak{a}_{i j}=0$ for $i>m$ or $j>m$, we shall say that $\mathfrak{b}^{\nabla}$ has order $\leq 2 m$.

Lemma 3.21. Let $P \in \operatorname{Diff}^{m}(M ; E, G)$ and $Q \in \operatorname{Diff}^{m}(M ; F, G)$, where $G$ is a Hermitian vector bundle. Then $\mathfrak{b}(u \otimes \bar{w}):=(P u, Q w)_{G}$ is a $\nabla$-bidifferential operator $\mathcal{C}_{c}^{\infty}(M \times M ; E \otimes \bar{F}) \rightarrow \mathcal{C}_{c}^{\infty}(M)$ of order $\leq 2 m$ with smooth coefficients. If $P$ and $Q$ have $\mathcal{C}_{b}^{\infty}$-coefficients, then $\mathfrak{b}$ will also have $\mathcal{C}_{b}^{\infty}$-coefficients.

Proof. We can assume $P=a \nabla^{i}$ and $Q=b \nabla^{j}$, by linearity and by the definition of $\nabla$-differential operators.

Recall that dvol denotes the volume form on $M$ associated to the metric.

Definition 3.22. The sesquilinear form

$$
B_{\mathfrak{b}}^{\nabla}(u, w):=\int_{M} \mathfrak{b}^{\nabla}(u \otimes \bar{w}) \mathrm{dvol}
$$

is called the Dirichlet form associated to $\mathfrak{b}^{\nabla}$. It has $\mathcal{C}_{\mathrm{b}}^{\infty}$-coefficients if $\mathfrak{b}^{\nabla}$ has and it has the same order as $\mathfrak{b}^{\nabla}$. The induced map $P_{\mathfrak{b}}^{\nabla}: \mathcal{C}_{\mathrm{c}}^{\infty}(M ; E) \rightarrow \mathcal{C}_{\mathrm{c}}^{\infty}(M ; F)^{*}$,

$$
\left\langle P_{\mathfrak{b}}^{\nabla} u, \bar{w}\right\rangle=B_{\mathfrak{b}}^{\nabla}(u, w), \quad u \in \mathcal{C}_{\mathrm{c}}^{\infty}(M ; E), w \in \mathcal{C}_{\mathrm{c}}^{\infty}(M ; F),
$$

is called the $\nabla$-differential operator in divergence form associated to $\mathfrak{b}^{\nabla}$ (or to $B_{\mathfrak{b}}^{\nabla}$ ). If $\mathfrak{a}_{i j}=0$ if $i>m$ or $j>m$, then we shall say that $P_{\mathfrak{b}}^{\nabla}$ has order $\leq 2 m$.

We shall see in the last part of this section that $P_{\mathfrak{b}}^{\nabla}$ is indeed a $\nabla$-differential operator. We shall continue to use the notation of Remark 3.20. In particular, $\mathfrak{b}^{\nabla}$ will be a $\nabla$-bidifferential operator. 
Lemma 3.23. Let $m \in \mathbb{N}$ be such that $\mathfrak{a}_{i j} \in \mathcal{C}_{b}^{\infty}\left(M ; \operatorname{Hom}\left(T^{* \otimes i} M \otimes E ; T^{* \otimes j} M \otimes F\right)\right)$, for all $i$ and $j$ and $a_{i j}=0$ if $i>m$ or $j>m$. Then $B_{\mathfrak{b}}^{\nabla}$ extends to a continuous, sesquilinear map

$$
B_{\mathfrak{b}}^{\nabla}: H_{\nabla}^{m}(M ; E) \times H_{\nabla}^{m}(M ; F) \rightarrow \mathbb{C} .
$$

Similarly, $P_{\mathfrak{b}}^{\nabla}$ extends to a continuous map

$$
P_{\mathfrak{b}}^{\nabla}: H_{\nabla}^{m}(M ; E) \rightarrow H_{\nabla}^{m}(M ; F)^{*} .
$$

Remark 3.24. It is clear that all of the following sets are vector spaces:

(1) The set $b i$-Diff ${ }_{\nabla}^{2 m}(M ; E, F)$ of $\nabla$-bidifferential operators $\mathfrak{b}^{\nabla}: \mathcal{C}_{\mathrm{c}}^{\infty}(M \times$ $M ; E \otimes \bar{F}) \rightarrow \mathcal{C}_{\mathrm{c}}^{\infty}(M)$ of order $\leq 2 m$ (with smooth coefficients).

(2) The set $b i$-Diff ${ }_{b, \nabla}^{2 m}(M ; E, F)$ of $\nabla$-bidifferential operators $\mathfrak{b}^{\nabla} \in b i$-Diff ${ }^{\mu}(M ; E, F)$ with $\mathcal{C}_{\mathrm{b}}^{\infty}$-coefficients.

(3) The set of Dirichlet forms $B_{\mathfrak{b}}^{\nabla}$ (with smooth coefficients) associated to $\mathfrak{b}^{\nabla} \in$ $b i$-Diff ${ }^{2 m}(M ; E, F)$.

(4) The set of Dirichlet forms $B_{\mathfrak{b}}^{\nabla}$ associated to $\mathfrak{b}^{\nabla} \in b i$-Diff ${ }_{b}^{\mu}(M ; E, F)$ (thus with $\mathcal{C}_{\mathrm{b}}^{\infty}$-coefficients).

(5) The set of order $\leq 2 m$ differential operators $P_{\mathfrak{b}}^{\nabla}$ in divergence form (with smooth coefficients) associated to $\mathfrak{b}^{\nabla} \in b i$-Diff ${ }^{\mu}(M ; E, F)$.

(6) The set of order $\leq 2 m$ differential operators $P_{\mathfrak{b}}^{\nabla}$ in divergence form associated to $\mathfrak{b}^{\nabla} \in b i$-Diff ${ }_{b}^{\mu}(M ; E, F)$ (thus with $\mathcal{C}_{\mathrm{b}}^{\infty}$-coefficients).

The coefficients $\mathfrak{a}_{i j}$ in the canonical form for $\mathfrak{b}^{\nabla}$ (see Remark 3.18) are not unique (except for $i+j \leq 1$ ). So when we say that one of the above objects has $\mathcal{C}_{\mathrm{b}}^{\infty}$-coefficients, we mean that we can choose the coefficients $\mathfrak{a}_{i j}$ to be in $\mathcal{C}_{\mathrm{b}}^{\infty}$.

Proposition 3.25. A linear map $\mathfrak{b}^{\text {mix }}: \mathcal{C}_{c}^{\infty}(M \times M ; E \otimes \bar{F}) \rightarrow \mathcal{C}_{c}^{\infty}(M)$ is a $\nabla$-bidifferential operator of order $\leq 2 m$ if, and only if, it is a linear combination of maps of the form $u \otimes \bar{w} \rightarrow(P u, Q w)_{G}$, where $P \in \operatorname{Diff}^{m}(M ; E, G)$ and $Q \operatorname{Diff}^{m}(M ; F, G)$. If $\mathfrak{b}$ has $\mathcal{C}_{b}^{\infty}$-coefficients, then we can choose $P$ and $Q$ to also have $\mathcal{C}_{b}^{\infty}$-coefficients.

Proof. This follows from definitions.

Proposition 3.26. Let $A \in \mathcal{C}^{\infty}(M ; \operatorname{End}(E)), A^{*}=-A$, and $\widetilde{\nabla}=\nabla+A$. Let also $p \in[1, \infty]$ and $\mu \in \mathbb{N}$. We then have the following:

(i) $b i-\operatorname{Diff}_{\nabla}^{\mu}(M ; E, F)=b i-\operatorname{Diff}_{\widetilde{\nabla}}^{\mu}(M ; E, F)$.

(ii) If $A \in \mathcal{C}_{b}^{\infty}(M ; \operatorname{End}(E))$, then $b i-\operatorname{Diff}_{b, \nabla}^{\mu}(M ; E, F)=b i-\operatorname{Diff}_{b, \widetilde{\nabla}}^{\mu}(M ; E, F)$.

Proof. This follows with arguments similar to those for Proposition 3.4.

Let us now introduce mixed $\nabla$-bidifferential operators. Recall that $\operatorname{Diff}^{m}(M ; E, G)$ denotes the set of mixed differential operators $\mathcal{C}_{\mathrm{c}}^{\infty}(M ; F) \rightarrow \mathcal{C}_{\mathrm{c}}^{\infty}(M ; G)$.

Definition 3.27. A linear map $\mathfrak{b}^{\text {mix }}: \mathcal{C}_{\mathrm{c}}^{\infty}(M \times M ; E \otimes \bar{F}) \rightarrow \mathcal{C}_{\mathrm{c}}^{\infty}(M)$ is a mixed bidifferential operator of order $\leq 2 m$ if it is a linear combination of maps of the form $u \otimes \bar{w} \rightarrow(P u, Q w)_{G}$, where $P \in \widetilde{\operatorname{Diff}}^{m}(M ; E, G)$ and $Q \in \widetilde{\operatorname{Diff}}^{m}(M ; F, G)$. If $P$ and $Q$ have $\mathcal{C}_{\mathrm{b}}^{\infty}$-coefficients, then we shall say that $\mathfrak{b}$ will also have $\mathcal{C}_{\mathrm{b}}^{\infty}$-coefficients.

Let us assume now (FFC) and derive then the equality of the space of $\nabla$ bidifferential operators and that of mixed bidifferential operators, in analogy with the corresponding result for differential operators. 
Proposition 3.28. Let $m \in \mathbb{N}$ and assume $M$ satisfies ( $F F C$ ).

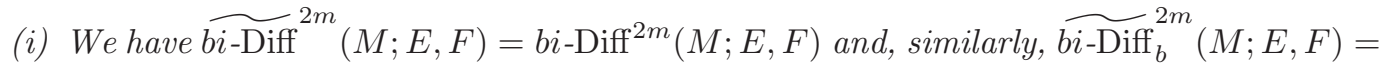
$b i$-Diff ${ }_{b}^{2 m}(M ; E, F)$.

(ii) Let $Z_{1}, Z_{2}, \ldots, Z_{N} \in \mathcal{W}_{b}(M)$ be Fréchet systems of generators for $\mathcal{W}_{b}(M)$ of Remark 3.8, then $\widetilde{\operatorname{Diff}}^{2 m}(M ; E, F)$ is linearly generated by sesqui-linear maps of the form

$$
u \otimes \bar{w} \rightarrow\left(a \nabla_{X_{1}} \nabla_{X_{2}} \ldots \nabla_{X_{r}}, b \nabla_{X_{r+1}} \nabla_{X_{r+2}} \ldots \nabla_{X_{r+s}}\right)_{G},
$$

where $r \leq m, X_{1}, X_{2}, \ldots, X_{r+s} \in\left\{Z_{1}, Z_{2}, \ldots, Z_{N}\right\}, a \in \mathcal{C}^{\infty}(M ; \operatorname{Hom}(E, G))$, $b \in \mathcal{C}^{\infty}(M ; \operatorname{Hom}(F, G))$, and $G$ is an auxiliary vector bundle.

(iii) The analogous result holds for $\widetilde{\text { bi-Diff }}_{b}^{2 m}(M ; E, F)$ with $a \in \mathcal{C}_{b}^{\infty}(M ; \operatorname{Hom}(E, G))$ and $b \in \mathcal{C}_{b}^{\infty}(M ; \operatorname{Hom}(F, G))$.

Proof. This follows from Proposition 3.4 and Lemma 3.21.

Proposition 3.29. Assume $M$ satisfies the (FFC) condition and let $\left(Z_{j}\right), 1 \leq j \leq$ $N$, be a Fréchet generating system for $\mathcal{W}_{b}(M)$.

(i) bi-Diff ${ }^{2 m}(M ; E, F)$ is linearly generated by sesquilinear maps of the form $u \otimes$ $\bar{w} \rightarrow\left(a \nabla_{Z_{k_{1}}}^{E} \ldots \nabla_{Z_{k_{r}}}^{E} u, b \nabla_{Z_{j_{1}}}^{E} \ldots \nabla_{Z_{j_{s}}}^{E}\right)_{G}$, where $1 \leq k_{1} \leq k_{2} \leq \ldots \leq k_{r} \leq N$, $1 \leq j_{1} \leq j_{2} \leq \ldots \leq j_{s} \leq N, r, s \leq m$, and $a \in \mathcal{C}^{\infty}(M ; \operatorname{Hom}(E ; G))$ and $b \in \mathcal{C}^{\infty}(M ; \operatorname{Hom}(F ; G))$.

Let us assume also that $E \rightarrow M$ and $F \rightarrow M$ have totally bounded curvature. Then

(ii) bi-Diff ${ }_{b}^{2 m}(M ; E, F)$ is linearly generated by sesquilinear maps $\mathfrak{b}$ of the form $\mathfrak{b}(u \otimes \bar{w})=\left(a \nabla_{Z_{k_{1}}}^{E} \ldots \nabla_{Z_{k_{r}}}^{E} u, b \nabla_{Z_{j_{1}}}^{E} \ldots \nabla_{Z_{j_{s}}}^{E}\right)_{G}$, where $1 \leq k_{1} \leq k_{2} \leq \ldots \leq$ $k_{r} \leq N, 1 \leq j_{1} \leq j_{2} \leq \ldots \leq j_{s} \leq N, r, s \leq m$, and $a \in \mathcal{C}_{b}^{\infty}(M ; \operatorname{Hom}(E ; G))$ and $b \in \mathcal{C}_{b}^{\infty}(M ; \operatorname{Hom}(F ; G))$.

Proof. This follows from Corollary 3.14 and Lemma 3.21.

We conclude with the following proposition.

Proposition 3.30. Assume that $M$ satisfies the ( $F F C$ ) condition and let $m \in \mathbb{N}$ and $\mathfrak{b} \in b i-\operatorname{Diff}^{2 m}(M ; E, F)$. Then the restriction $P$ of $P_{\mathfrak{b}}^{\nabla}$,

$$
P_{\mathfrak{b}}^{\nabla}: H_{\nabla}^{m}(M ; E) \rightarrow H_{\nabla}^{-m}(M ; F),
$$

obtained from the restriction $H_{\nabla}^{m}(M ; F)^{m} \rightarrow H_{\nabla}^{-m}(M ; F)$, is a $\nabla$-differential operator of order $\leq 2 m$, that is $P \in \operatorname{Diff}_{b}^{2 m}(M ; E, F)$.

Proof. By linearity, we can assume that $\mathfrak{b}(u \otimes \bar{w})=\left(a \nabla^{i} u, \nabla^{j} w\right)_{T * \otimes j M \otimes F}$, where $a \in \mathcal{C}_{\mathrm{b}}^{\infty}\left(M ; \operatorname{Hom}\left(T^{* \otimes i} M \otimes E ; T^{* \otimes j} M \otimes F\right)\right.$. Then $P=\left(\nabla^{j}\right)^{*} a \nabla^{i} \in \operatorname{Diff}_{b}^{2 m}(M ; E, F)$ by Proposition 3.10(ii).

Corollary 3.31. Assume that $M$ satisfies $(F F C)$ and let $m \in \mathbb{N}$ and $\mathfrak{b} \in b i$-Diff ${ }^{2 m}(M ; E, F)$ with respect to the metric $g$ of $M$. Let $\rho, f_{0}: M \rightarrow(0, \infty)$ be admissible weights with respect to the metric $g_{0}=\rho^{-2} g$. Then the restriction $P$ of $P_{\mathfrak{b}}^{\nabla}$ to weighted Sobolev spaces,

$$
P_{\mathfrak{b}}^{\nabla}: f_{0} H_{\nabla^{L C}, \rho}^{m}(M ; E) \rightarrow \frac{1}{f_{0}} H_{\nabla^{L C}, \rho}^{-m}(M ; F),
$$


obtained from the restriction $f_{0} H_{\nabla^{L C}, \rho}^{m}(M ; F)^{m} \rightarrow \frac{1}{f_{0}} H_{\nabla^{L C}, \rho}^{-m}(M ; F)$, is a $\nabla$-differential operator of order $\leq 2 m$, that is $P \in \operatorname{Diff}_{b}^{2 m}(M ; E, F)$.

Proof. The result follows from Proposition 3.30 and Corollary 3.12 .

\section{REFERENCES}

[1] Aldana, C., Carron, G., And Tapie, S. $a_{\infty}$ weights and compactness of conformal metrics under $l^{n / 2}$ curvature bounds. ArXiv preprint 1810.05387, 2018.

[2] Amann, H. Function spaces on singular manifolds. Math. Nachr. 286, 5-6 (2013), 436-475.

[3] Amann, H. Parabolic equations on uniformly regular Riemannian manifolds and degenerate initial boundary value problems. In Recent developments of mathematical fluid mechanics, Adv. Math. Fluid Mech. Birkhäuser/Springer, Basel, 2016, pp. 43-77.

[4] Amann, H. Cauchy problems for parabolic equations in Sobolev-Slobodeckii and Hölder spaces on uniformly regular Riemannian manifolds. J. Evol. Equ. 17, 1 (2017), 51-100.

[5] Ammann, B., And Gross E, N. $L^{p}$-spectrum of the Dirac operator on products with hyperbolic spaces. Calc. Var. Partial Differential Equations 55, 5 (2016), 127-163.

[6] Ammann, B., Grosse, N., And Nistor, V. The strong Legendre condition and the wellposedness of mixed Robin-Dirichlet problems on manifolds with bounded geometry. Preprint arXiv: 1810.06926.

[7] Ammann, B., Grosse, N., And Nistor, V. Well-posedness of the Laplacian on manifolds singular spaces.

[8] Ammann, B., Grosse, N., And Nistor, V. Analysis and boundary value problems on singular domains: an approach via bounded geometry. C. R. Math. Acad. Sci. Paris 357, 6 (2019), 487-493.

[9] Ammann, B., Grosse, N., and Nistor, V. Well-posedness of the Laplacian on manifolds with boundary and bounded geometry. Math. Nachr. 292, 6 (2019), 1213-1237.

[10] Ammann, B., Grosse, N., And Nistor, V. Analysis and boundary value problems on domains with oscillating singular points. Preprint, 2020.

[11] Ammann, B., Ionescu, A. D., And Nistor, V. Sobolev spaces on Lie manifolds and regularity for polyhedral domains. Doc. Math. 11 (2006), 161-206 (electronic).

[12] Ammann, B., And Nistor, V. Weighted Sobolev spaces and regularity for polyhedral domains. Comput. Methods Appl. Mech. Engrg. 196, 37-40 (2007), 3650-3659.

[13] Aronszajn, N., and Milgram, A. N. Differential operators on Riemannian manifolds. Rend. Circ. Mat. Palermo (2) 2 (1953), 266-325.

[14] Aubin, T. Espaces de Sobolev sur les variétés riemanniennes. Bull. Sci. Math. (2) 100, 2 (1976), 149-173.

[15] Aubin, T. Some nonlinear problems in Riemannian geometry. Springer Monographs in Mathematics. Springer-Verlag, Berlin, 1998.

[16] Bacuta, C., Nistor, V., AND Zikatanov, L. Improving the rate of convergence of high-order finite elements on polyhedra. I. A priori estimates. Numer. Funct. Anal. Optim. 26, 6 (2005), 613-639.

[17] Bacuta, C., Nistor, V., and Zikatanov, L. Improving the rate of convergence of high-order finite elements on polyhedra. II. Mesh refinements and interpolation. Numer. Funct. Anal. Optim. 28, 7-8 (2007), 775-824.

[18] Besse, A. L. Einstein manifolds. Classics in Mathematics. Springer-Verlag, Berlin, 2008. Reprint of the 1987 edition.

[19] Bourgain, J., Brezis, H., And Mironescu, P. Another look at Sobolev spaces. In Optimal control and partial differential equations. IOS, Amsterdam, 2001, pp. 439-455.

[20] Browder, F. E. On the spectral theory of elliptic differential operators. I. Math. Ann. 142 (1960/61), 22-130.

[21] BăCuţă, C., Mazzucato, A., Nistor, V., and Zikatanov, L. Interface and mixed boundary value problems on $n$-dimensional polyhedral domains. Doc. Math. 15 (2010), 687-745.

[22] Carron, G. Formes harmoniques $L^{2}$ sur les variétés non-compactes. Rend. Mat. Appl. (7) 21, 1-4 (2001), 87-119.

[23] Costabel, M., Dauge, M., And Nicaise, S. Analytic regularity for linear elliptic systems in polygons and polyhedra. Math. Models Methods Appl. Sci. 22, 8 (2012), 1250015, 63. 
[24] Dauge, M. Elliptic boundary value problems on corner domains, vol. 1341 of Lecture Notes in Mathematics. Springer-Verlag, Berlin, 1988. Smoothness and asymptotics of solutions.

[25] Disconzi, M., Ifrim, M., and Tataru, D. The relativistic euler equations with a physical vacuum boundary: Hadamard local well-posedness, rough solutions, and continuation criterion. https://arxiv.org/abs/2007.05787, 2020.

[26] Disconzi, M., Shao, Y., And Simonett, G. Some remarks on uniformly regular riemannian manifolds. Preprint arXiv:1410.8627.

[27] Golénia, S., and Moroianu, S. Spectral analysis of magnetic Laplacians on conformally cusp manifolds. Ann. Henri Poincaré 9, 1 (2008), 131-179.

[28] GolÉnia, S., And Moroianu, S. The spectrum of Schrödinger operators and Hodge Laplacians on conformally cusp manifolds. Trans. Amer. Math. Soc. 364, 1 (2012), 1-29.

[29] Gounoue F, G.-F. A remake on the Bourgain-Brezis-Mironescu characterization of Sobolev spaces. arXiv:2008.07631v2 (2020), 1-24.

[30] Grosse, N., and Nistor, V. Uniform Shapiro-Lopatinski Conditions and Boundary Value Problems on Manifolds with Bounded Geometry. Potential Anal. 53, 2 (2020), 407-447.

[31] Grosse, N., And Schneider, C. Sobolev spaces on Riemannian manifolds with bounded geometry: general coordinates and traces. Math. Nachr. 286, 16 (2013), 1586-1613.

[32] Hebey, E. Sobolev spaces on Riemannian manifolds, vol. 1635 of Lecture Notes in Mathematics. Springer-Verlag, Berlin, 1996.

[33] Hebey, E., And Robert, F. Sobolev spaces on manifolds. In Handbook of global analysis. Elsevier Sci. B. V., Amsterdam, 2008, pp. 375-415, 1213.

[34] Hörmander, L. The analysis of linear partial differential operators. III. Classics in Mathematics. Springer, Berlin, 2007. Pseudo-differential operators, Reprint of the 1994 edition.

[35] Iftimie, V., Măntoiu, M., and Purice, R. Magnetic pseudodifferential operators. Publ. Res. Inst. Math. Sci. 43, 3 (2007), 585-623.

[36] Jost, J. Partial differential equations, second ed., vol. 214 of Graduate Texts in Mathematics. Springer, New York, 2007.

[37] Kohr, M., and Nistor, V. Sobolev spaces and $\nabla$-differential operators on manifolds II: Christoffel polynomials. Preprint, 2020.

[38] Kohr, M., And Wendland, W. Variational approach for the Stokes and Navier-Stokes systems with nonsmooth coefficients in Lipschitz domains on compact Riemannian manifolds. Calc. Var. Partial Differential Equations, 3-4 (2018), 57-165.

[39] Kondrat'Ev, V. A. Boundary value problems for elliptic equations in domains with conical or angular points. Transl. Moscow Math. Soc. 16 (1967), 227-313.

[40] Kozlov, V., Maz'ya, V., and Rossmann, J. Spectral problems associated with corner singularities of solutions to elliptic equations, vol. 85 of Mathematical Surveys and Monographs. American Mathematical Society, Providence, RI, 2001.

[41] Lions, J.-L., And Magenes, E. Non-homogeneous boundary value problems and applications. Vol. I. Springer-Verlag, New York-Heidelberg, 1972. Translated from the French by P. Kenneth, Die Grundlehren der mathematischen Wissenschaften, Band 181.

[42] Mazzucato, A., And Nistor, V. Mapping properties of heat kernels, maximal regularity, and semi-linear parabolic equations on noncompact manifolds. J. Hyperbolic Differ. Equ. 3, 4 (2006), 599-629.

[43] Milnor, J., And Stasheff, J. Characterstic classes, vol. 76 of Annals of Mathematics Studies. Princeton, 1974.

[44] Mitrea, D., Mitrea, I., Mitrea, M., and Taylor, M. The Hodge-Laplacian, vol. 64 of De Gruyter Studies in Mathematics. De Gruyter, Berlin, 2016. Boundary value problems on Riemannian manifolds.

[45] Moroinun, S. Weyl laws on open manifolds. Math. Ann. 340, 1 (2008), 1-21.

[46] Nazarov, S. A., And Plamenevsky, B. A. Elliptic problems in domains with piecewise smooth boundaries, vol. 13 of de Gruyter Expositions in Mathematics. Walter de Gruyter \& Co., Berlin, 1994.

[47] Nguyen, H.-M., Pinamonti, A., Squassina, M., and Vecchi, E. Some characterizations of magnetic Sobolev spaces. Complex Var. Elliptic Equ. 65, 7 (2020), 1104-1114.

[48] Peetre, J. Une caractérisation abstraite des opérateurs différentiels. Math. Scand. 7 (1959), $211-218$.

[49] Rudin, W. Functional analysis. 2nd edition. McGraw-Hill, Inc., New York, 1991.

[50] Seeley, R. T. Singular integrals on compact manifolds. Amer. J. Math. 81 (1959), 658-690. 
[51] Strichartz, R. Analysis of the Laplacian on the complete Riemannian manifold. J. Funct. Anal. 52, 1 (1983), 48-79.

[52] Taylor, M. Partial differential equations I. Basic theory, second ed., vol. 115 of Applied Mathematical Sciences. Springer, New York, 2011.

[53] Triebel, H. Characterizations of function spaces on a complete Riemannian manifold with bounded geometry. Math. Nachr. 130 (1987), 321-346.

[54] Triebel, H. Theory of function spaces. Modern Birkhäuser Classics. Birkhäuser/Springer Basel AG, Basel, 2010. Reprint of 1983 edition [MR0730762], Also published in 1983 by Birkhäuser Verlag [MR0781540].

Faculty of Mathematics and Computer Science, Babeş-Bolyai University, 1 M. Kogălniceanu Str., 400084 Cluj-Napoca, Romania

Email address: mkohr@math.ubbcluj.ro

Université de Lorraine, UFR MiM, Ile du Saulcy, CS 50128, 57045 METZ, France and

Inst. Math. Romanian Acad. PO BOX 1-764, 014700 Bucharest Romania

Email address: victor.nistor@univ-lorraine.fr 